\title{
Chemoselective Synthesis of Aryloxy-substituted Phthalocyanines
}

\author{
Taniyuki Furuyama ${ }^{*}, 1,2$, Kazuya Maeda ${ }^{1}$, Hajime Maeda ${ }^{1}$ and Masahito Segi ${ }^{1}$ \\ ${ }^{I}$ Graduate School of Natural Science and Technology, Kanazawa University, Kakuma-machi, Kanazawa, \\ 920-1192, Japan(tfuruyama@se.kanazawa-u.ac.jp) \\ ${ }^{2}$ Japan Science and Technology Agency (JST)-PRESTO, 4-1-8 Honcho, Kawaguchi, Saitama, 332-0012, \\ Japan
}

\section{Supporting information}

\section{Table of Contents}

Crystallographic Data Correction and Measurement of ${ }^{1} \mathrm{O}_{2}$ Generation Efficiency $\quad \mathrm{S}-2$

Additional Experimental Results $\quad$ S-5

Copies of the NMR Spectra of Studied Compounds $\quad$ S-15

$\begin{array}{ll}\text { Full Computational Details } & \text { S-28 }\end{array}$

$\begin{array}{ll}\text { References } & \text { S-38 }\end{array}$ 


\section{Crystallographic data collection}

Data collection for $\mathbf{1 a}$ and $\mathbf{3 b} \cdot\left(\mathrm{CHCl}_{3}\right)_{2}$ was carried out on a Bruker APEXIII CCD diffractometer with Bruker Helios multilayered confocal mirror monochromatized $\mathrm{CuK \alpha}$ radiation $(\lambda=1.54178 \AA)$ at $183^{\circ} \mathrm{C}$. The structures were solved by a direct method (SIR2004) ${ }^{1}$ and refined using a full-matrix least square technique (SHELXL-2014). ${ }^{2}$ Yadokari-XG 2009 software was used as a GUI for SHELXL-2014. ${ }^{3}$ All non-hydrogen atoms were refined anisotropically. Positions of all hydrogen atoms were calculated geometrically, and refined by applying riding models. CCDC-1922469 and 1922470 contains the supplementary crystallographic data. Their data can be obtained free of charge from Crystallographic Data Centre via www.ccdc.cam.ac.uk/data_request/cif.

\section{Measurement of ${ }^{1} \mathrm{O}_{2}$ generation efficiency}

Singlet oxygen quantum yields $\left(\Phi_{\Delta}\right)$ were determined in $\mathrm{CHCl}_{3}$ using a steady-state method ${ }^{4}$ with $\beta \beta$-octa-( $p$-tert-butylphenoxy) phthalocyaninato zinc $\left(\mathbf{S t d}-\mathbf{Z n P c}, \Phi_{\Delta}=0.73\right.$ in $\left.\mathrm{CHCl}_{3}{ }^{5}\right)$ as reference. 1,3-diphenylisobenzofuran (DPBF) was used as the chemical quencher for single oxygen in $\mathrm{CHCl}_{3}$. Irradiation was carried out with a halogen lamp (ALA-100, Asahi Spectra). A band path filter (680 nm, band width at 0.5 peak $=12.00 \mathrm{~nm}$, Asahi Spectra) was placed in the light beam path of the lamp. The light intensity was measured with an illuminometer (T-10A, KONICA MINOLTA) and adjusted to $1.5 \mathrm{~lx}$ at $680 \mathrm{~nm}$. Equation (1) was employed for the calculations:

$$
\Phi_{\Delta}=\Phi_{\Delta}^{\mathrm{Std}} \frac{R \cdot I_{\mathrm{abs}}^{\mathrm{Std}}}{R^{\mathrm{Std}} \cdot I_{\mathrm{abs}}}
$$

where $\Phi^{\mathrm{Std}}$ is the singlet oxygen quantum yield for the standard $\left(\Phi^{\mathrm{Std}}{ }_{\Delta}=0.73\right.$ in $\left.\mathrm{CHCl}_{3}\right) . R$ and $R$ Std are the DPBF photobleaching rates in the presence of the samples and standard, respectively. $I_{\mathrm{abs}}$ and $I_{\text {abs }}^{\text {Std }}$ are the rates of light absorption at $680 \mathrm{~nm}$ by the samples and standard, respectively. 
Table S1. Crystal data and structure refinement for $\mathbf{1 a}$

\begin{tabular}{|c|c|c|}
\hline Empirical formula & \multicolumn{2}{|l|}{$\mathrm{C}_{20} \mathrm{H}_{12} \mathrm{~N}_{2} \mathrm{O}_{2}$} \\
\hline Formula weight & \multicolumn{2}{|l|}{312.32} \\
\hline Temperature & \multicolumn{2}{|l|}{$90(2) \mathrm{K}$} \\
\hline Wavelength & \multicolumn{2}{|l|}{$1.54178 \AA$} \\
\hline Crystal system & \multicolumn{2}{|l|}{ Triclinic } \\
\hline Space group & \multicolumn{2}{|l|}{$P-1$} \\
\hline \multirow[t]{3}{*}{ Unit cell dimensions } & $a=8.8184(6) \AA$ & $\alpha=104.981(3)^{\circ}$ \\
\hline & $b=12.8640(8) \AA$ & $\beta=90.846(3)^{\circ}$ \\
\hline & $c=14.6760(10) \AA$ & $\gamma=101.054(4)^{\circ}$ \\
\hline Volume & $1574.78(18) \AA^{3}$ & \\
\hline$Z$ & 4 & \\
\hline Density (Calcd.) & $1.317 \mathrm{Mg} / \mathrm{m}^{3}$ & \\
\hline Absorption coefficient & $0.701 \mathrm{~mm}^{-1}$ & \\
\hline$F(000)$ & 648 & \\
\hline Crystal size & $0.200 \times 0.200 \times 0$. & \\
\hline Theta range for data collection & 3.124 to $66.488^{\circ}$ & \\
\hline Index ranges & $-10<=h<=9,-15<=$ & $<=l<=17$ \\
\hline Reflections collected & 11485 & \\
\hline Independent reflections & $5352[R($ int $)=0.02$ & \\
\hline Completeness to theta $=66.500^{\circ}$ & $96.3 \%$ & \\
\hline Refinement method & Full-matrix least-s & \\
\hline Data / restraints /parameters & $5352 / 0 / 517$ & \\
\hline Goodness-of-fit on $F^{2}$ & 1.101 & \\
\hline Final $R$ indices $[I>2 \operatorname{sigma}(I)]$ & $R_{1}=0.0438, w R_{2}=$ & \\
\hline$R$ indices (all data) & $R_{1}=0.0499, w R_{2}=$ & \\
\hline Largest diff. peak and hole & 0.251 and $-0.236 \mathrm{e}$ & \\
\hline CCDC No. & 1922469 & \\
\hline
\end{tabular}


Table S2. Crystal data and structure refinement for $\mathbf{3 b} \cdot\left(\mathrm{CHCl}_{3}\right)_{2}$

\begin{tabular}{|c|c|}
\hline Empirical formula & $\mathrm{C}_{90} \mathrm{H}_{68} \mathrm{Cl}_{6} \mathrm{~N}_{8} \mathrm{O}_{16}$ \\
\hline Formula weight & 1730.22 \\
\hline Temperature & $90(2) \mathrm{K}$ \\
\hline Wavelength & $1.54178 \AA$ \\
\hline Crystal system & Monoclinic \\
\hline Space group & $P 2_{1} / c$ \\
\hline \multirow[t]{3}{*}{ Unit cell dimensions } & $a=15.9706(5) \AA$ \\
\hline & $b=23.3689(7) \AA$ \\
\hline & $c=19.9594(6) \AA$ \\
\hline Volume & $7997.9(4) \AA^{3}$ \\
\hline$Z$ & 4 \\
\hline Density (Calcd.) & $1.437 \mathrm{Mg} / \mathrm{m}^{3}$ \\
\hline Absorption coefficient & $2.592 \mathrm{~mm}^{-1}$ \\
\hline$F(000)$ & 3576 \\
\hline Crystal size & $0.200 \times 0.200 \times 0.200 \mathrm{~mm}^{3}$ \\
\hline Theta range for data collection & 2.797 to $66.498^{\circ}$ \\
\hline Index ranges & $-18<=h<=18,-30<=k<=30,-23<=l<=23$ \\
\hline Reflections collected & 56584 \\
\hline Independent reflections & $14026[R($ int $)=0.0445]$ \\
\hline Completeness to theta $=66.500^{\circ}$ & $99.4 \%$ \\
\hline Refinement method & Full-matrix least-squares on $F^{2}$ \\
\hline Data / restraints / parameters & 14026 / 0 / 1089 \\
\hline Goodness-of-fit on $F^{2}$ & 1.030 \\
\hline Final $R$ indices $[I>2 \operatorname{sigma}(I)]$ & $R_{1}=0.0716, w R_{2}=0.1947$ \\
\hline$R$ indices (all data) & $R_{1}=0.0808, w R_{2}=0.2070$ \\
\hline Largest diff. peak and hole & 1.176 and -1.566 e. $\AA^{-3}$ \\
\hline CCDC No. & 1922470 \\
\hline
\end{tabular}




\section{Additional Experimental Results}

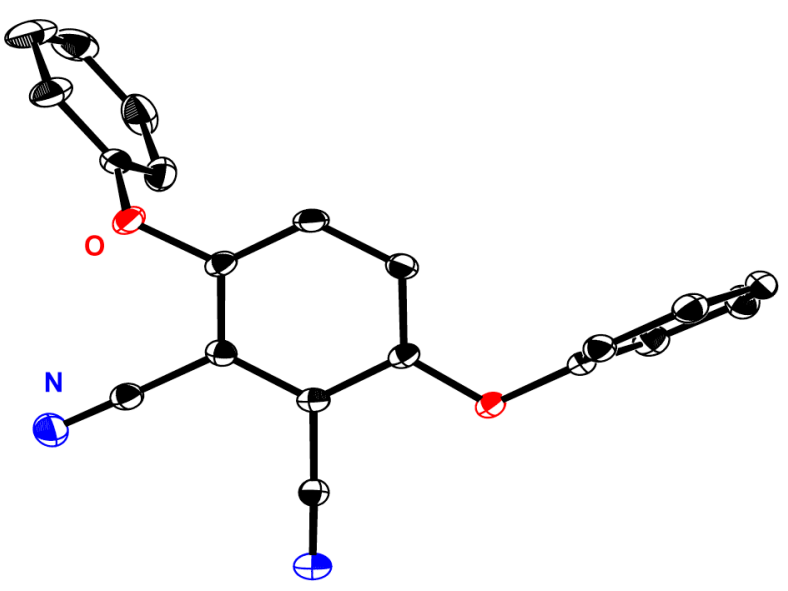

Figure S1. Molecular structure of 3,6-diphenoxyphthalonitrile (1a) with thermal ellipsoids at 50\% probability; hydrogen atoms have been omitted for clarity and only selected atoms have been labeled.

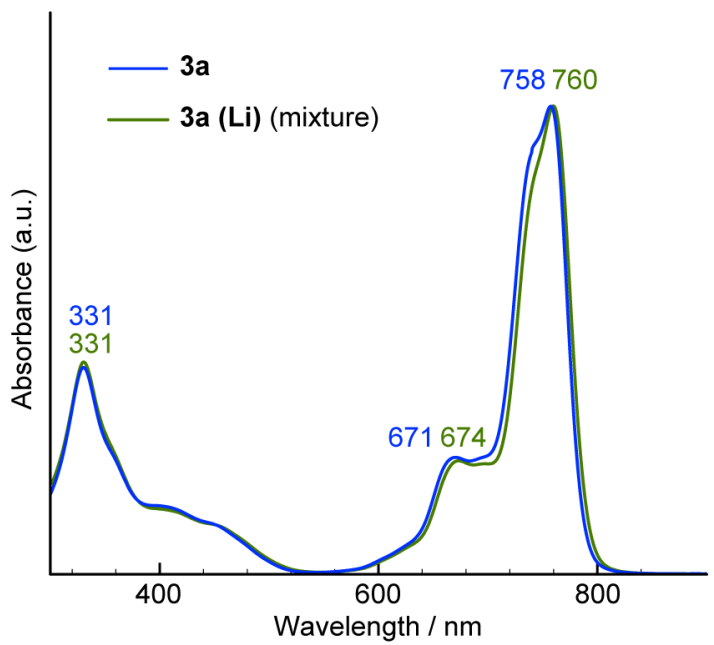

Figure S2. UV-vis-NIR absorption spectra of 3a and $\mathbf{3 a}\left(\mathbf{L i )}\right.$ in $\mathrm{CH}_{2} \mathrm{Cl}_{2}$. 


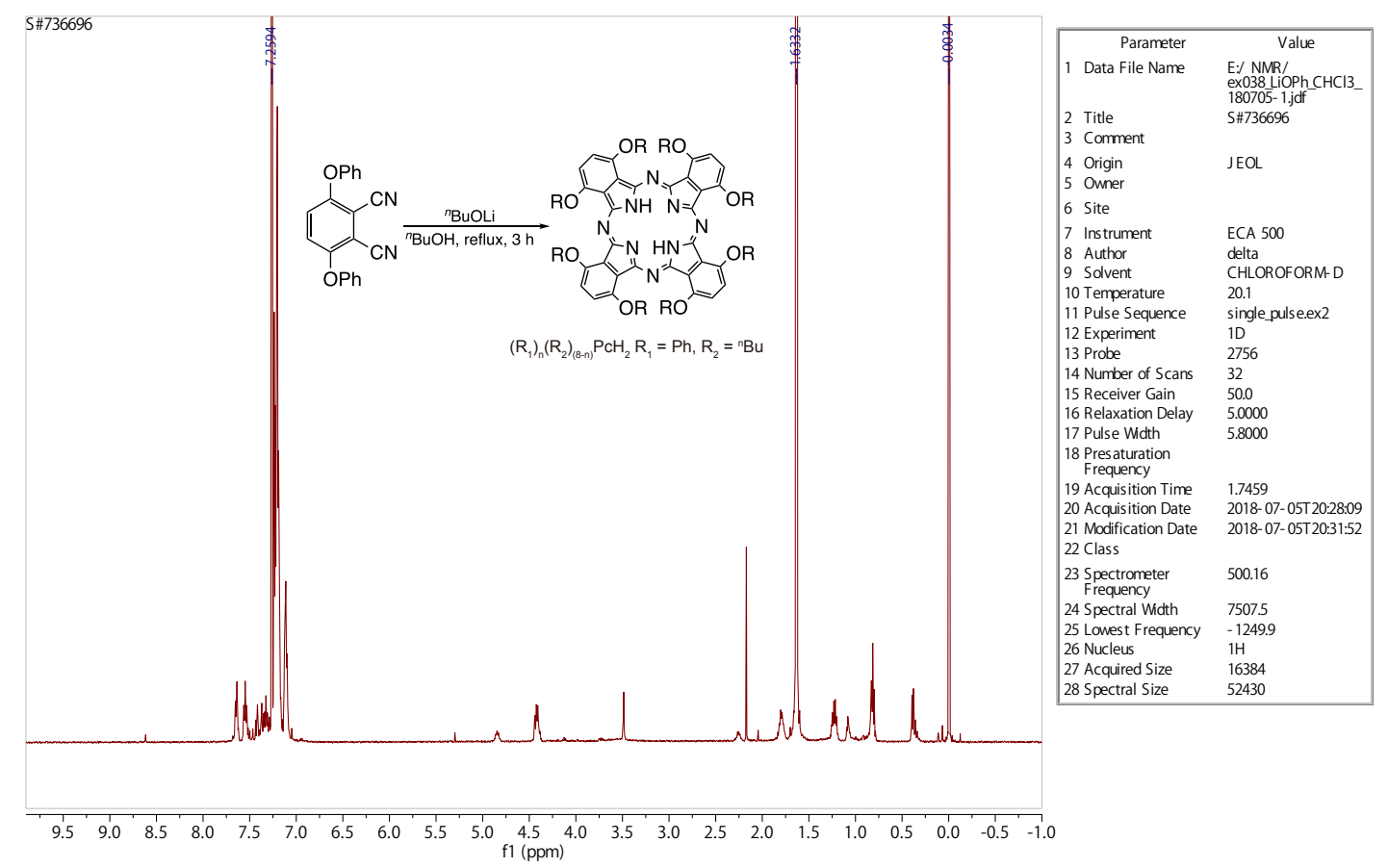

Figure S3. ${ }^{1} \mathrm{H}$ NMR spectrum of 3a (Li) (1a was treated under lithium method conditions; the detailed conditions are described in the inset of the spectrum) in $\mathrm{CDCl}_{3}$. The complex peak pattern indicates that pure 3a was not obtained.

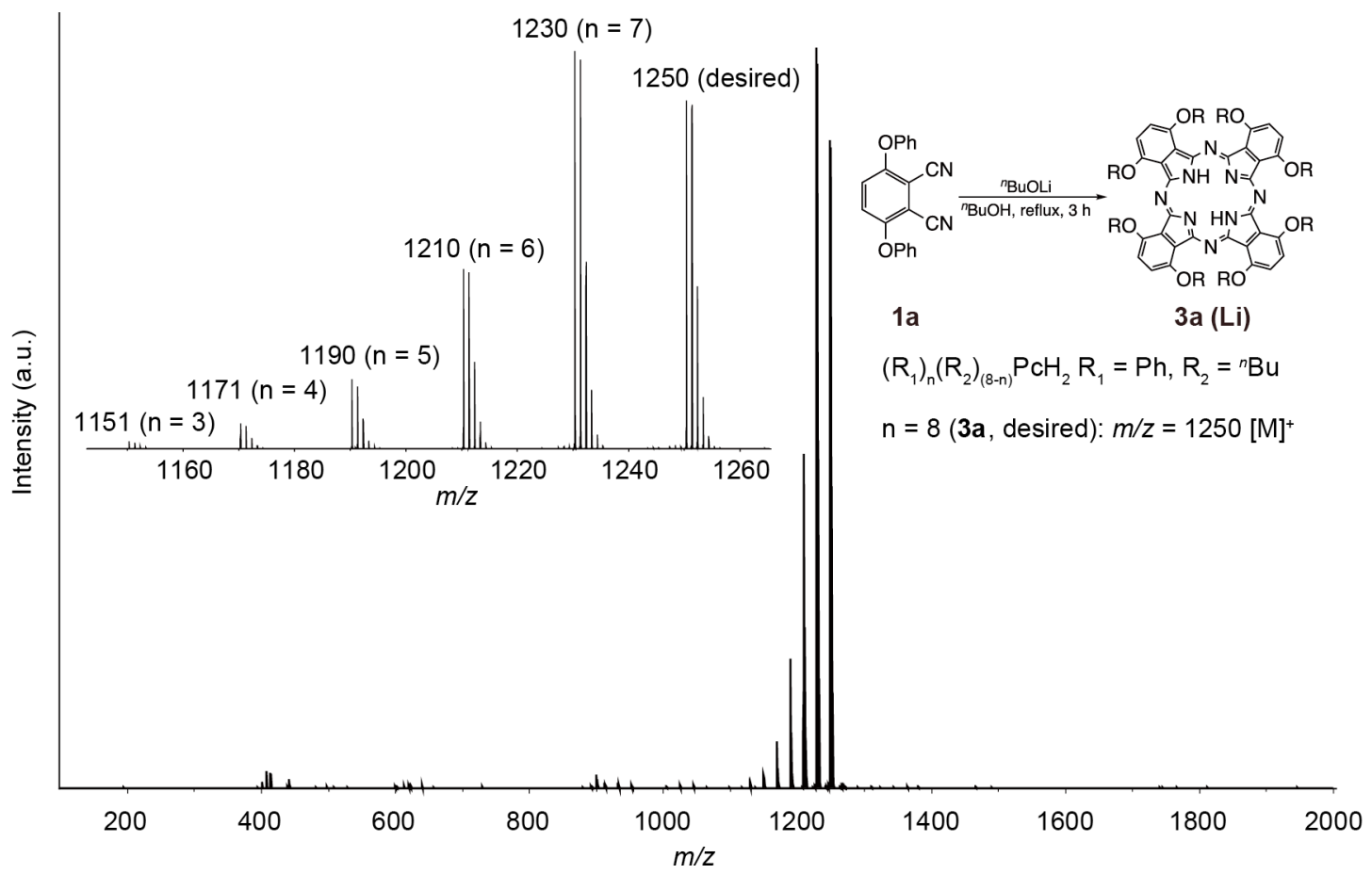

Figure S4. MALDI-FT-ICR-MS spectrum of 3a (Li); inset: magnified spectrum. 


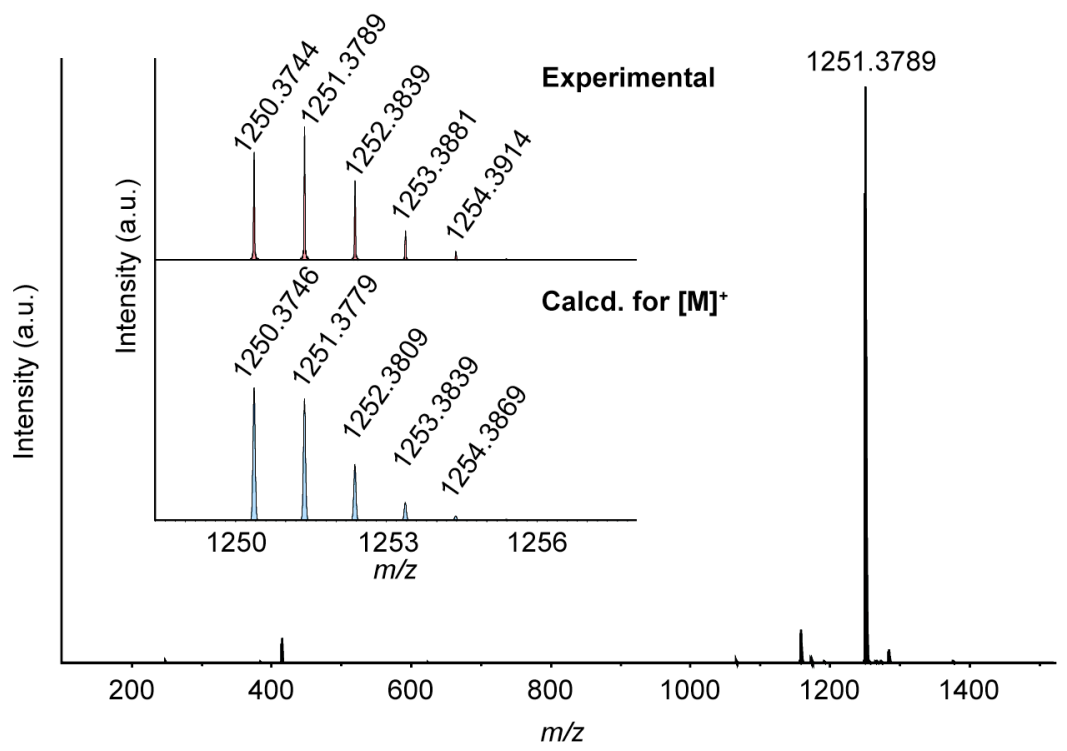

Figure S5. HR-MALDI-FT-ICR-MS spectrum of 3a; inset: magnified spectrum. 


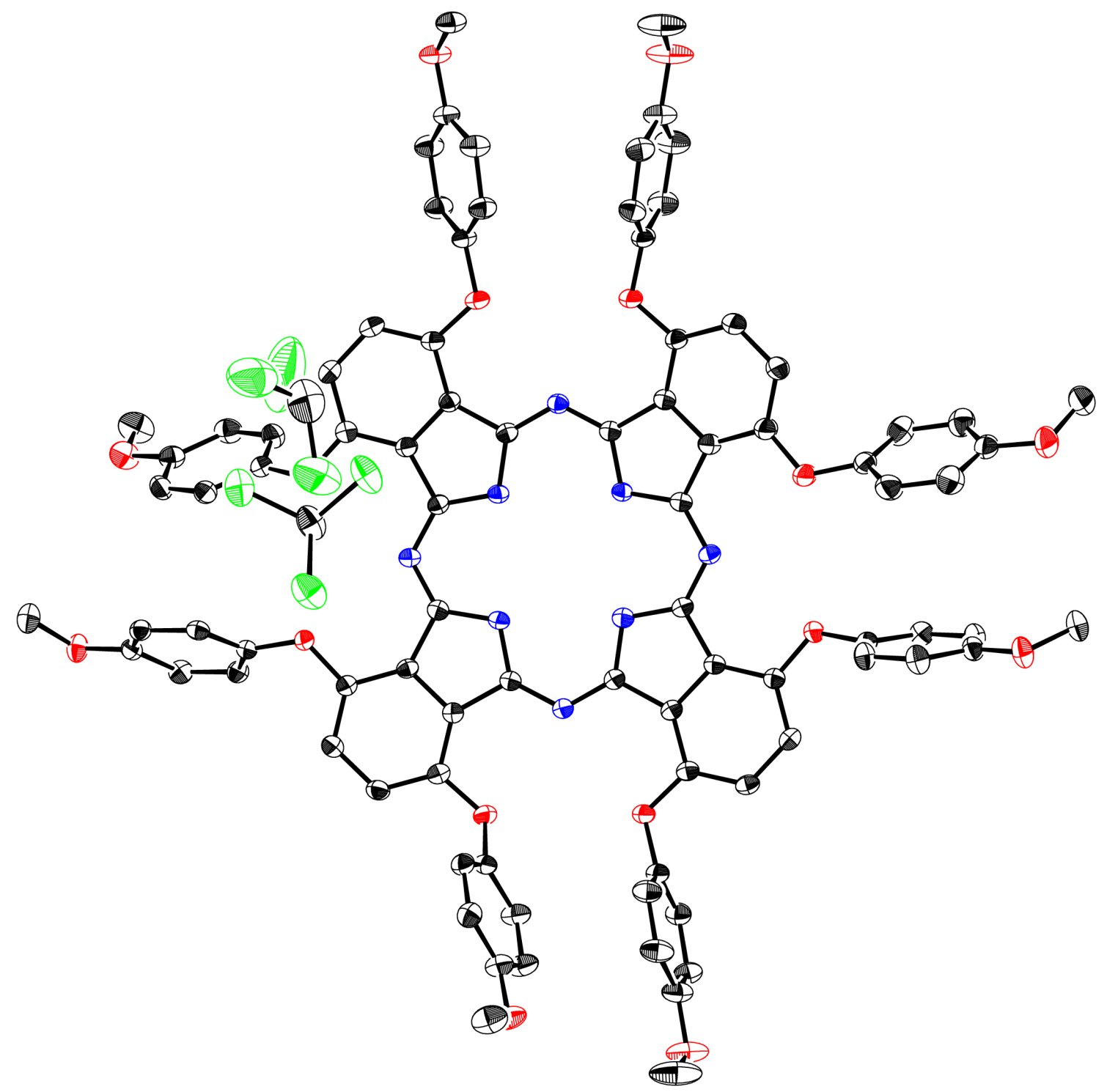

Figure S6. Thermal ellipsoid plot for the crystal structure $\mathbf{3 b}$ (two chloroform molecules contains in the unit cell) at $50 \%$ probability. Hydrogen atoms have been omitted for clarity. The single crystals were prepared by the recrystallization from the diffusion of hexane into a chloroform solution of $\mathbf{3 b}$. 


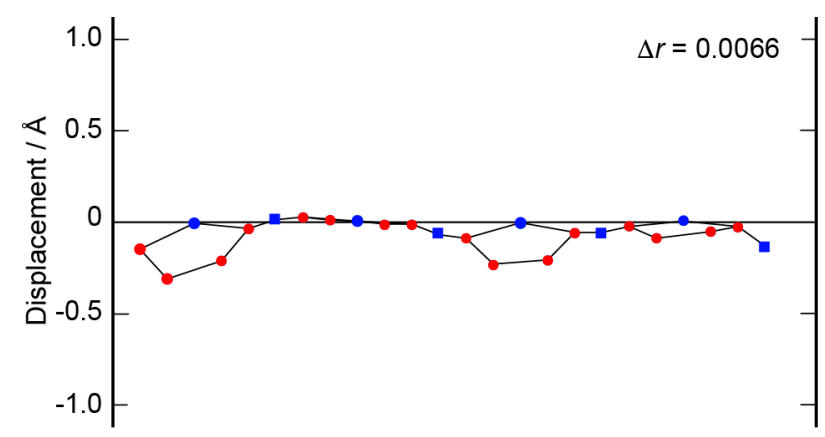

Figure S7. Skeletal deviation of the atoms from the $4 N$ mean plane for the crystallographic structure of 3b. Red circles indicate carbon atoms. Blue squares and circles indicate nitrogen atoms at meso-positions and coordinating nitrogen atoms, respectively.
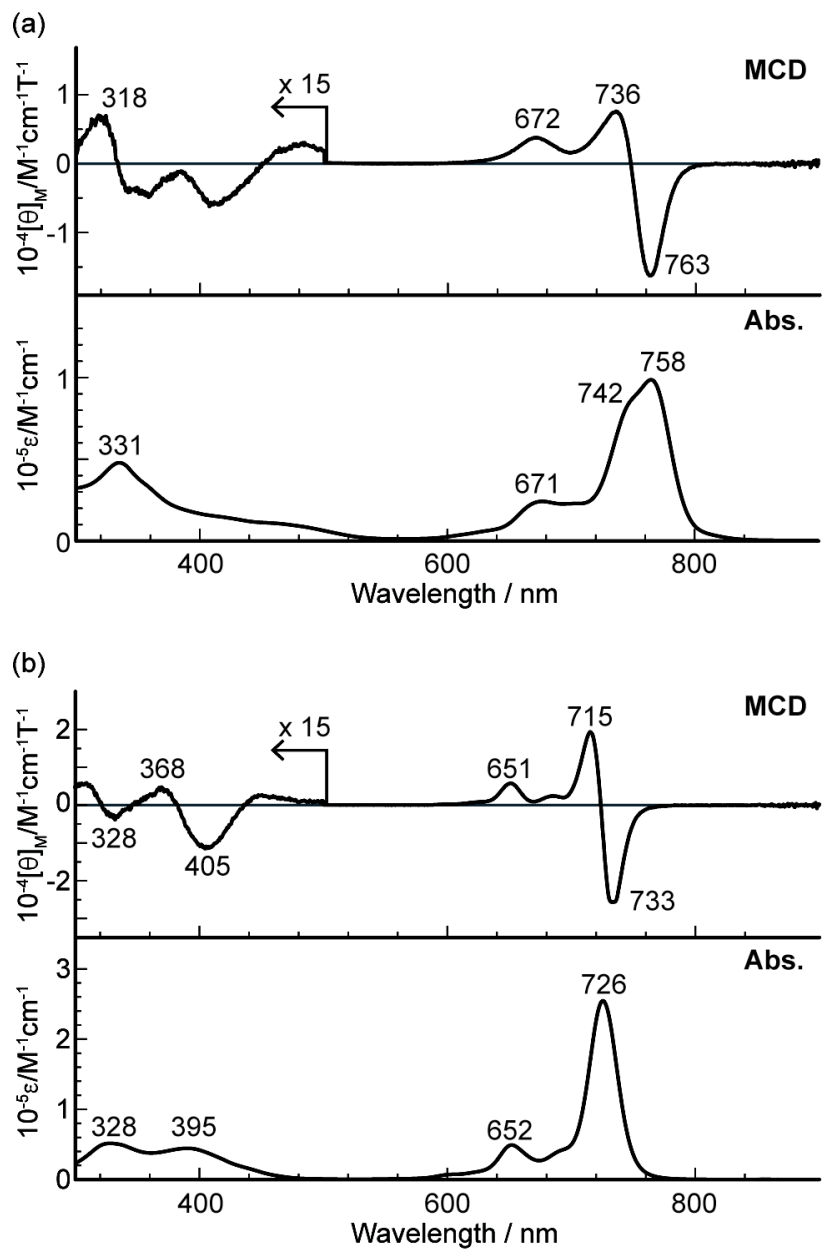

Figure S8. UV-vis-NIR absorption (bottom) and magnetic circular dichroism (MCD) (top) spectra of (a) 3a in $\mathrm{CH}_{2} \mathrm{Cl}_{2}$ and (b) $4 \mathbf{a}$ in THF. 


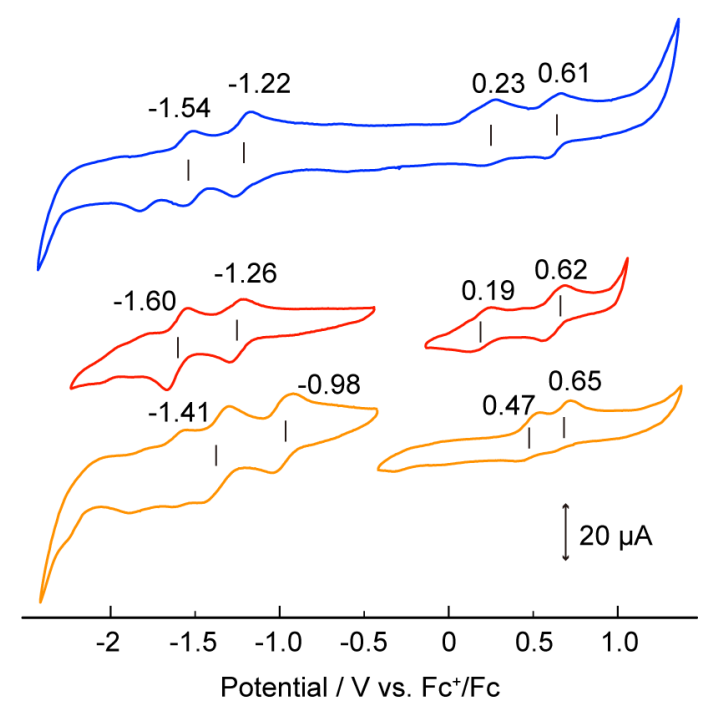

Figure S9. Cyclic voltammograms of 3a (blue), 3b (red), and 3d (orange) recorded from $1.0 \mathrm{mM}$ solutions of the analytes in $\left[{ }^{n} \mathrm{Bu}_{4} \mathrm{~N}\right] \mathrm{ClO}_{4} / \mathrm{CH}_{2} \mathrm{Cl}_{2}$. Ferrocene was used as the internal standard, whereby the $\mathrm{Fc} / \mathrm{Fc}^{+}$couple was set to $0 \mathrm{~V}$.

(a)

$E[\mathrm{eV}]$

Figure S10. Partial molecular energy diagram and orbitals (a) of 3a, as well as its calculated absorption spectra. Calculations were carried out at the B3LYP/6-31G* level of theory. 
(a)

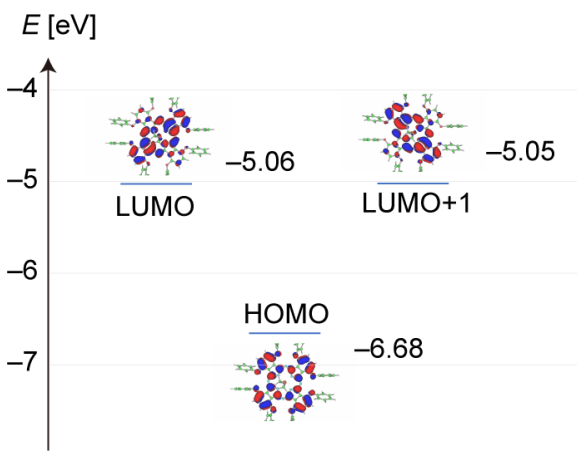

(b)

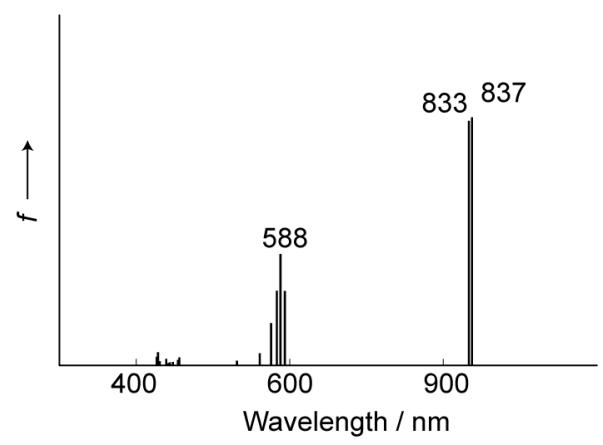

Figure S11. Partial molecular energy diagram and orbitals (a) of 8a' (the cation (Pc) part of 8a), as well as its calculated absorption spectra. Calculations were carried out at the B3LYP/6-31G* level of theory.

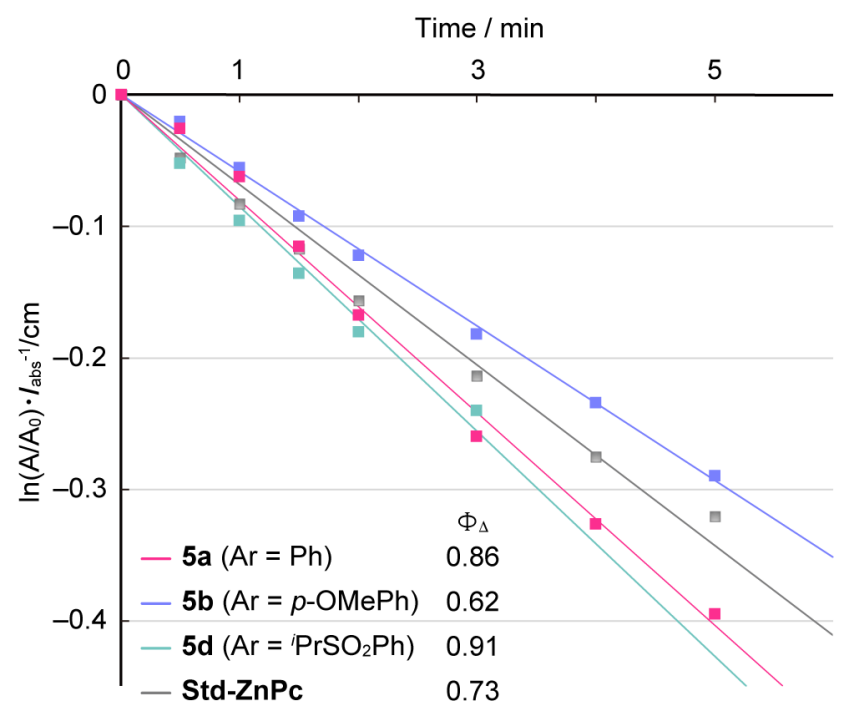

Figure S12. Comparison of the rate of decay of 1,3-diphenylisobenzofuran (DPBF) sensitized by Pcs in $\mathrm{CHCl}_{3}\left(\sim 5.0 \times 10^{-6} \mathrm{M}\right)$ as shown by the decrease in the absorbance at $415 \mathrm{~nm}$. The absorption coefficient was normalized by the rates of the absorption at $680 \mathrm{~nm}$. The singlet oxygen quantum yields were determined by using $\beta \beta$-octa-( $p$-tert-butylphenoxy) phthalocyaninato zinc (Std-ZnPc) as the reference; inset: Singlet oxygen quantum yields $\left(\Phi_{\Delta}\right)$ of the compounds. 


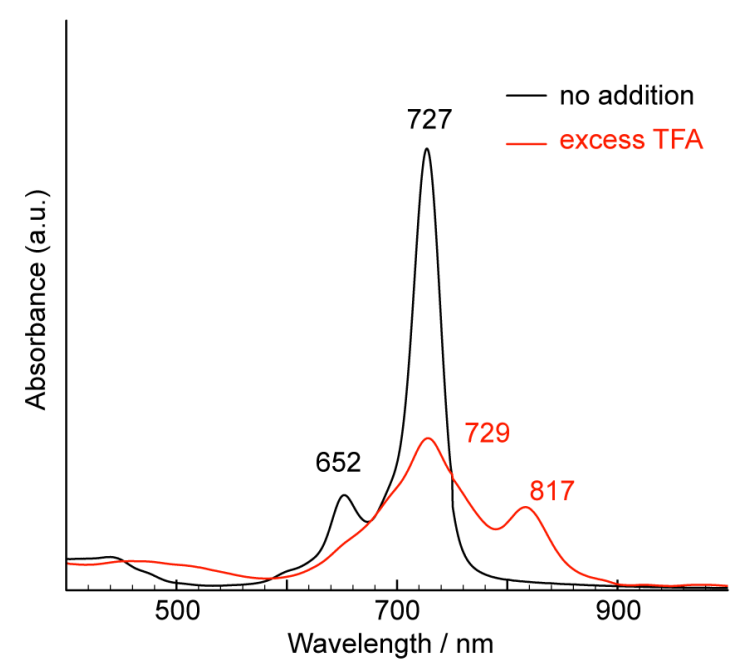

Figure S13. Absorption spectra of $6 \mathbf{a}\left([c]_{0}=5.0 \times 10^{-6} \mathrm{M}\right)$ in THF (black line) and after addition of $\mathrm{CF}_{3} \mathrm{COOH}$ (ca. 2000 eq.) to the solution (red line).
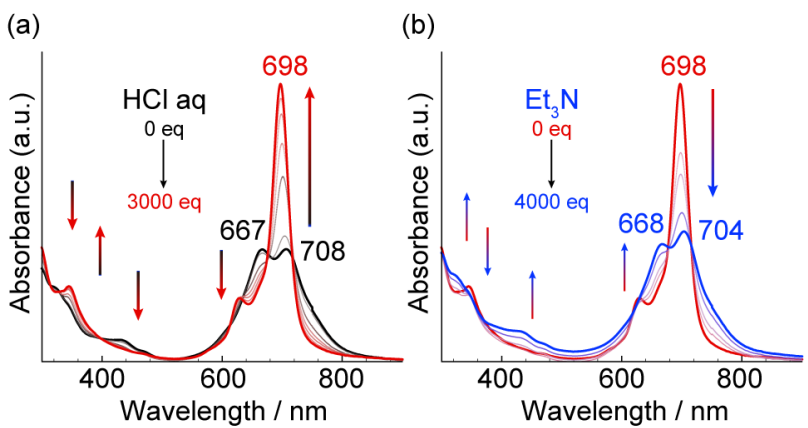

Figure S14. Absorption spectral changes of $6 \mathrm{e}$ in methanol $\left([c]_{0}=3.0 \times 10^{-6} \mathrm{M}\right)$ by (a) adding $\mathrm{HCl}(0-$ 3000 eq., black to red lines) in methanol, then (b) adding $\mathrm{Et}_{3} \mathrm{~N}$ (0-4000 eq., red to blue lines). 


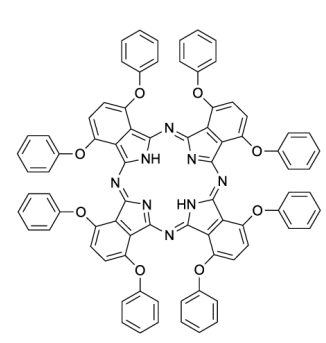

3a
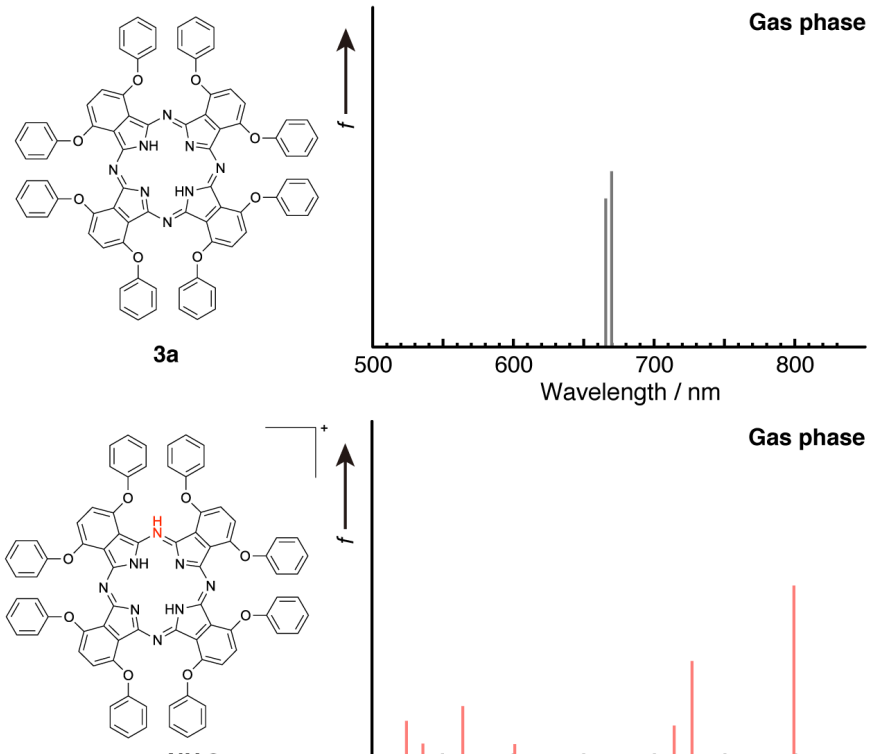

meso-NH 3a
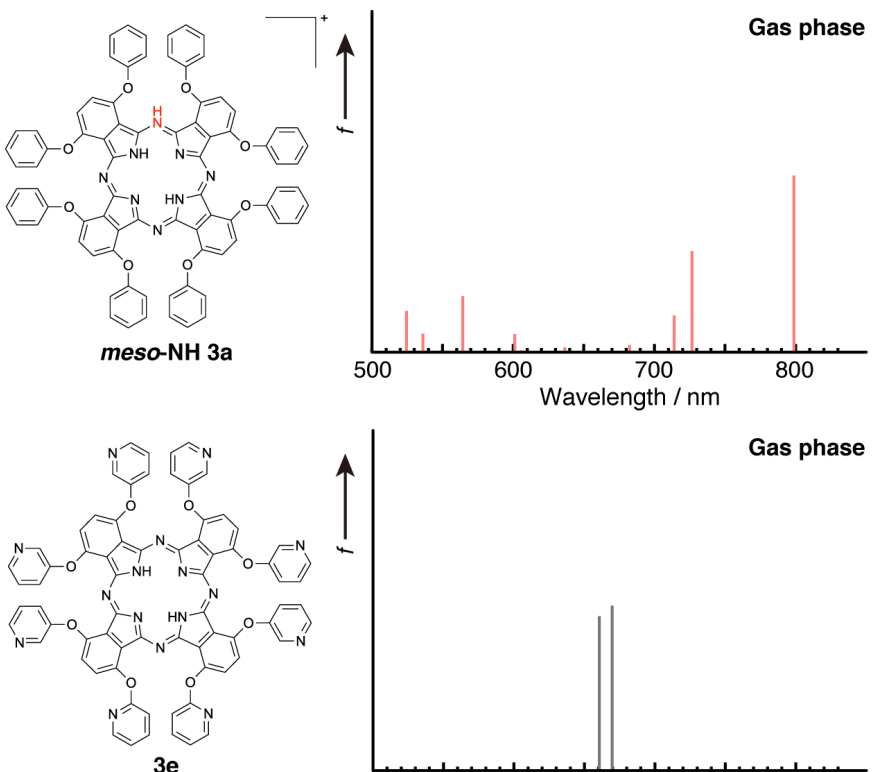

$3 e$

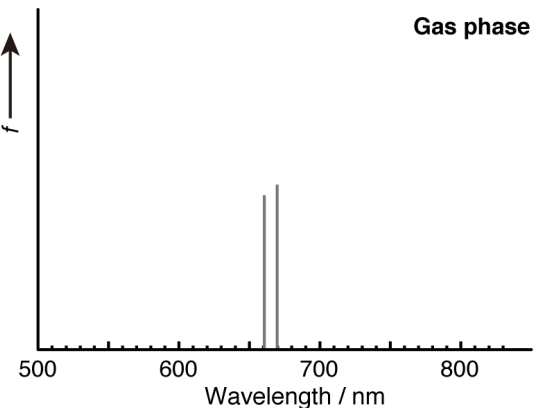

in methanol

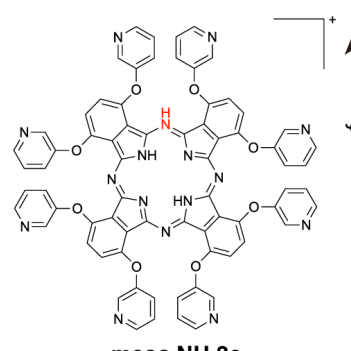

meso-NH $3 e$
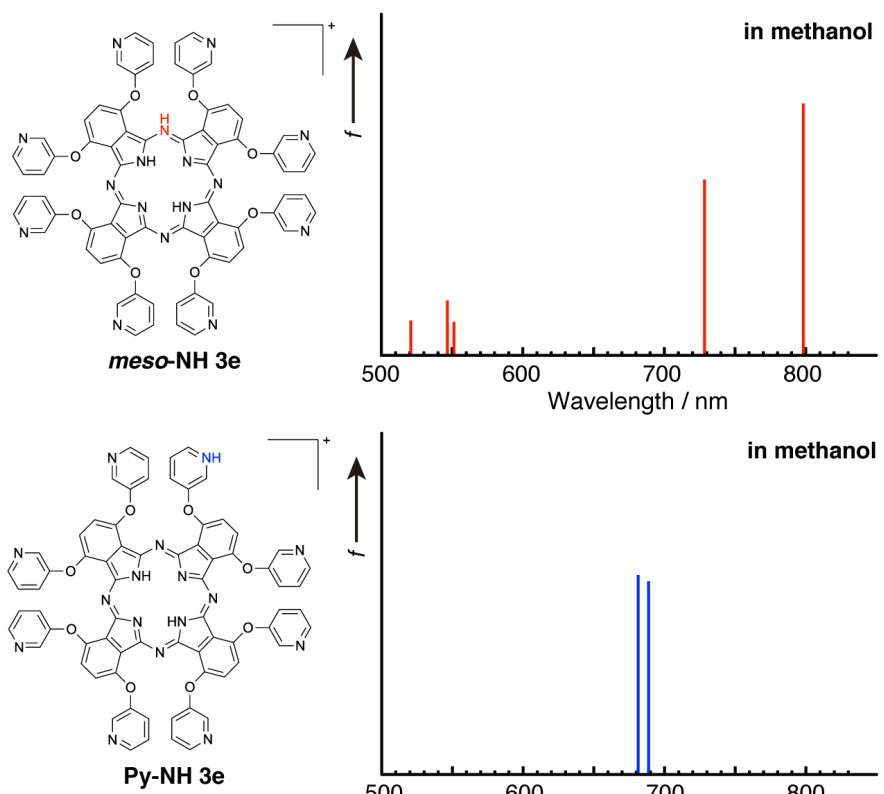

Py-NH 3e

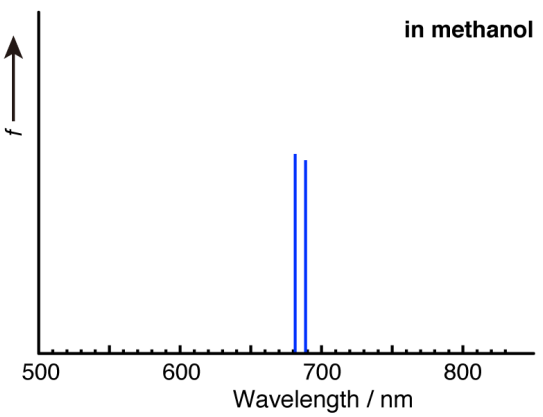

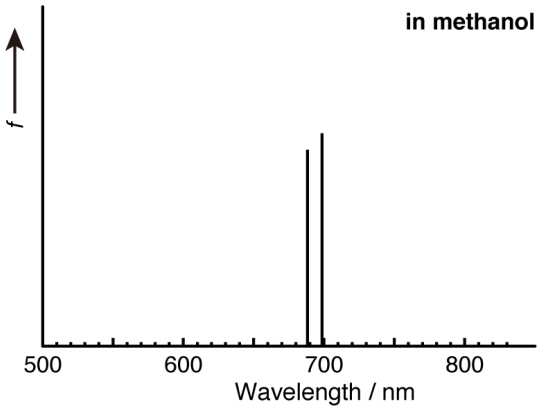
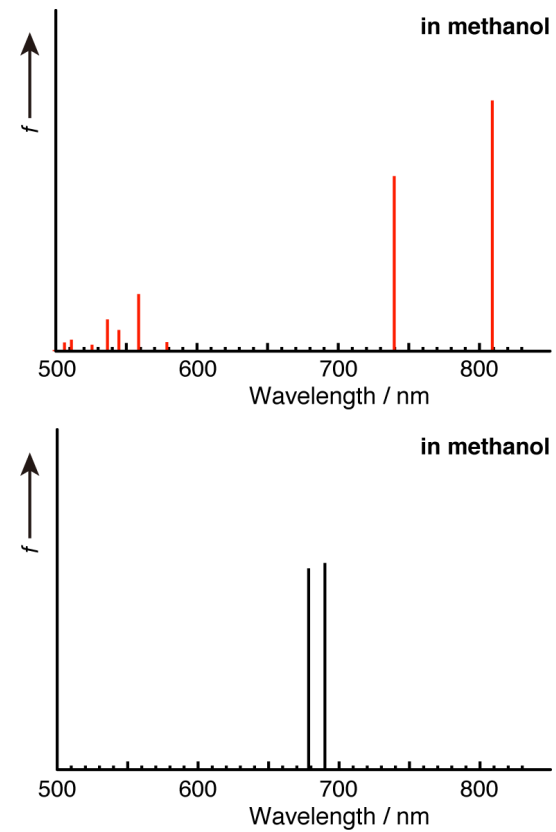

Figure S15. Calculated absorption spectra of 3a, 3e, and their protonated species. Calculations were carried out at the B3LYP/6-31G* level of theory, using the polarizable continuum model (PCM) that mimicked the solvation effect of methanol. 

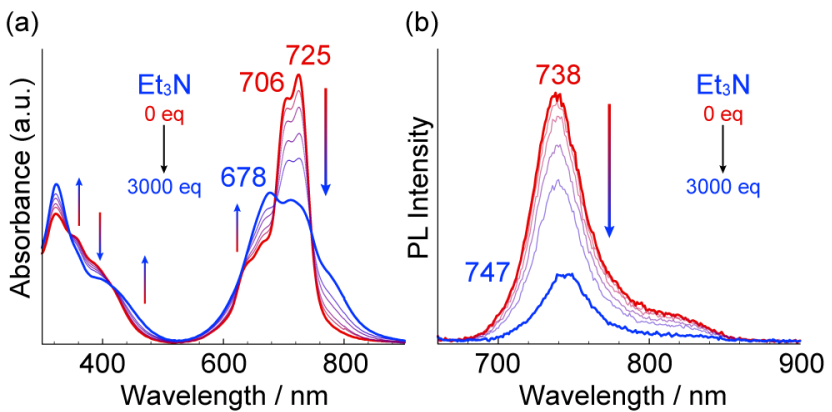

Figure S16. Spectral changes of 3e in methanol by adding $E t_{3} N$ (0-3000 eq.) in methanol, starting from 3e $\left([c]_{0}=3.0 \times 10^{-6} \mathrm{M}\right)$ and 2000 eq of $\mathrm{HCl}$ (red line; $c f$. Figure 4). (a) Absorption spectra. (b) Fluorescence spectra. 


\section{Copies of the NMR Spectra of Studied Compounds}

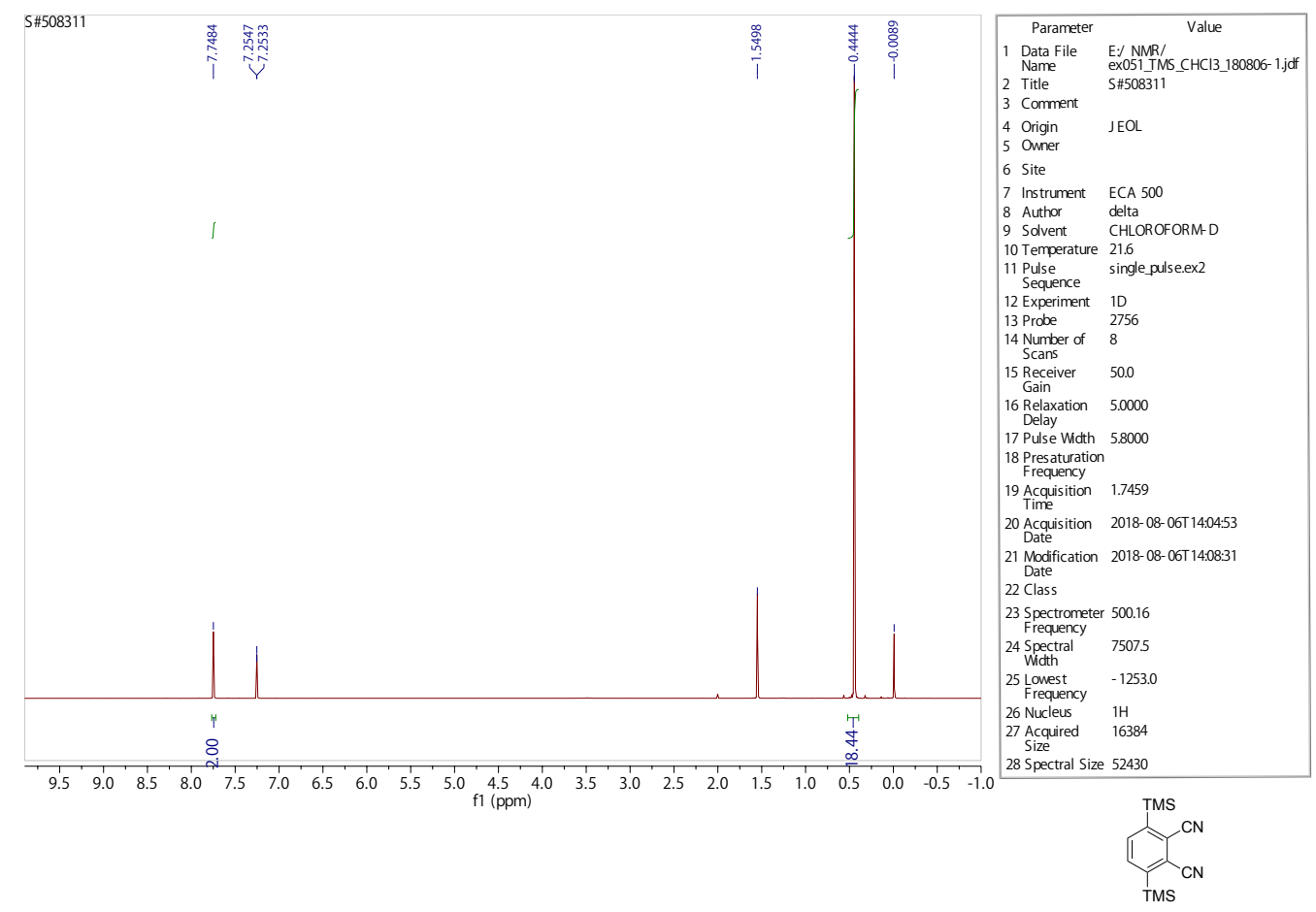

Figure S17. ${ }^{1} \mathrm{H}$ NMR spectra of 3,6-Bis(trimethylsilyl)phthalonitrile in $\mathrm{CDCl}_{3}$.

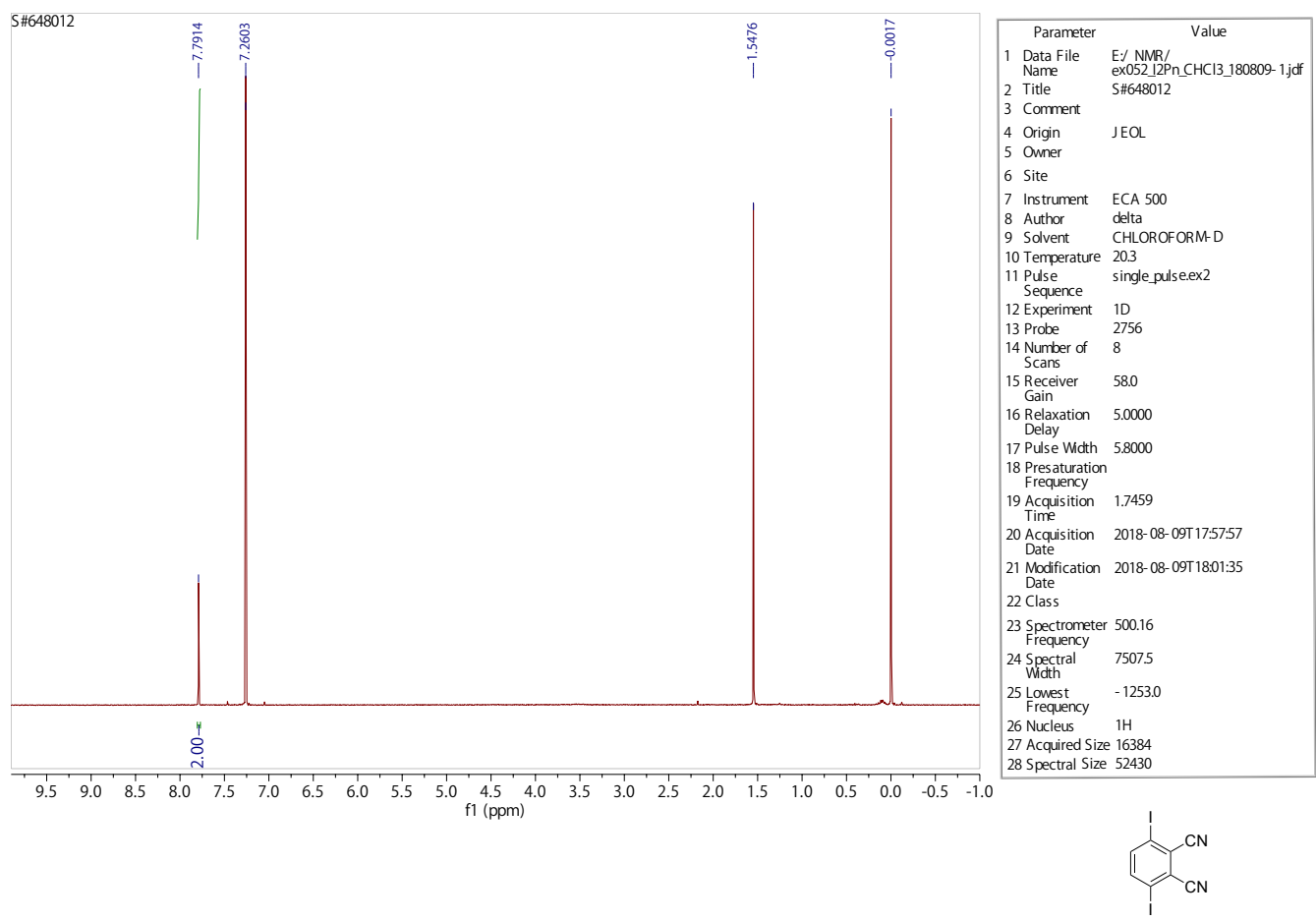

Figure S18. ${ }^{1} \mathrm{H}$ NMR spectra of 3,6-Diiodophthalonitrile in $\mathrm{CDCl}_{3}$. 

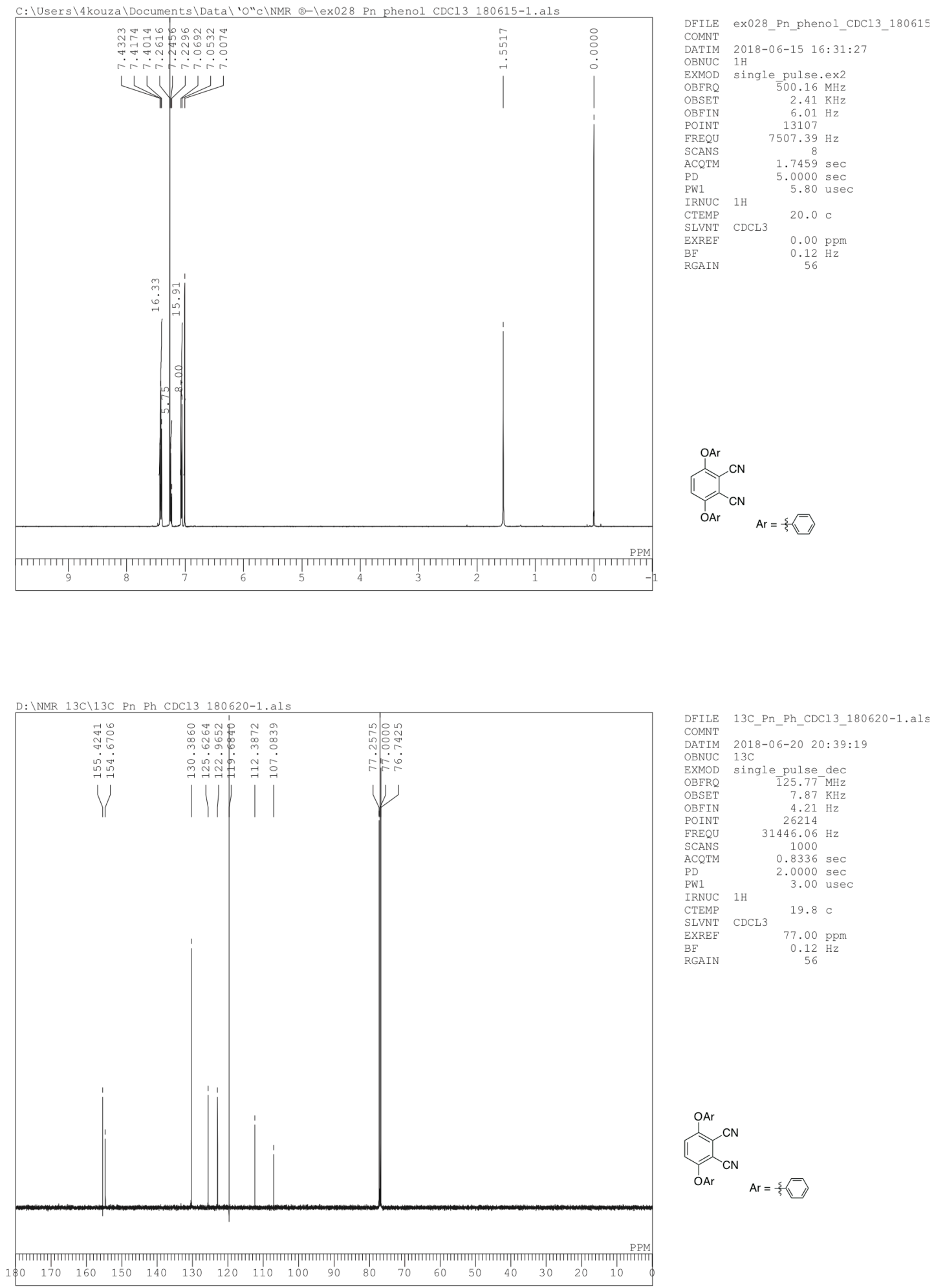

Figure S19. ${ }^{1} \mathrm{H}$ (top) and ${ }^{13} \mathrm{C}$ (bottom) NMR spectra of $1 \mathbf{a}$ in $\mathrm{CDCl}_{3}$. 

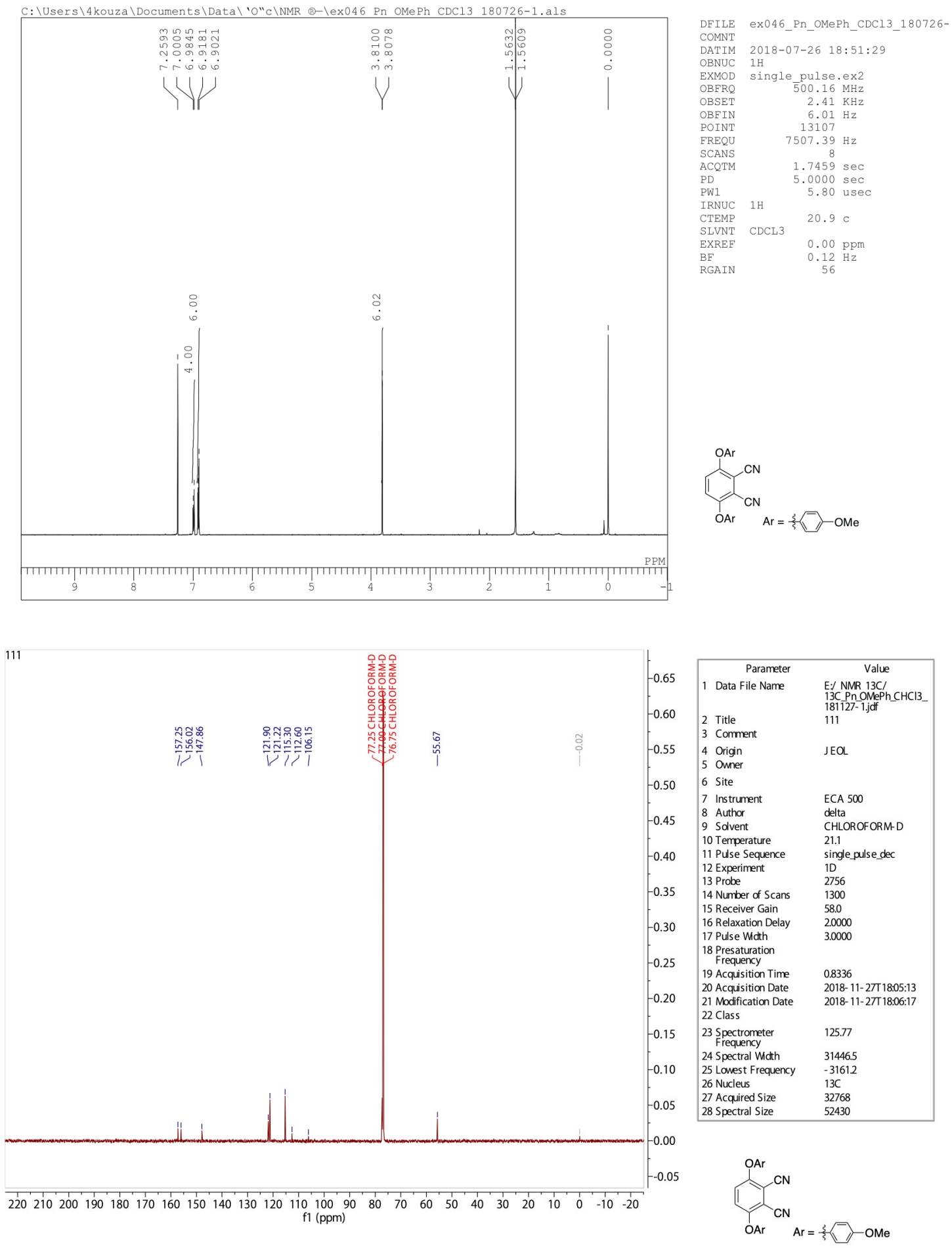

Figure S20. ${ }^{1} \mathrm{H}$ (top) and ${ }^{13} \mathrm{C}$ (bottom) NMR spectra of $\mathbf{1 b}$ in $\mathrm{CDCl}_{3}$. 

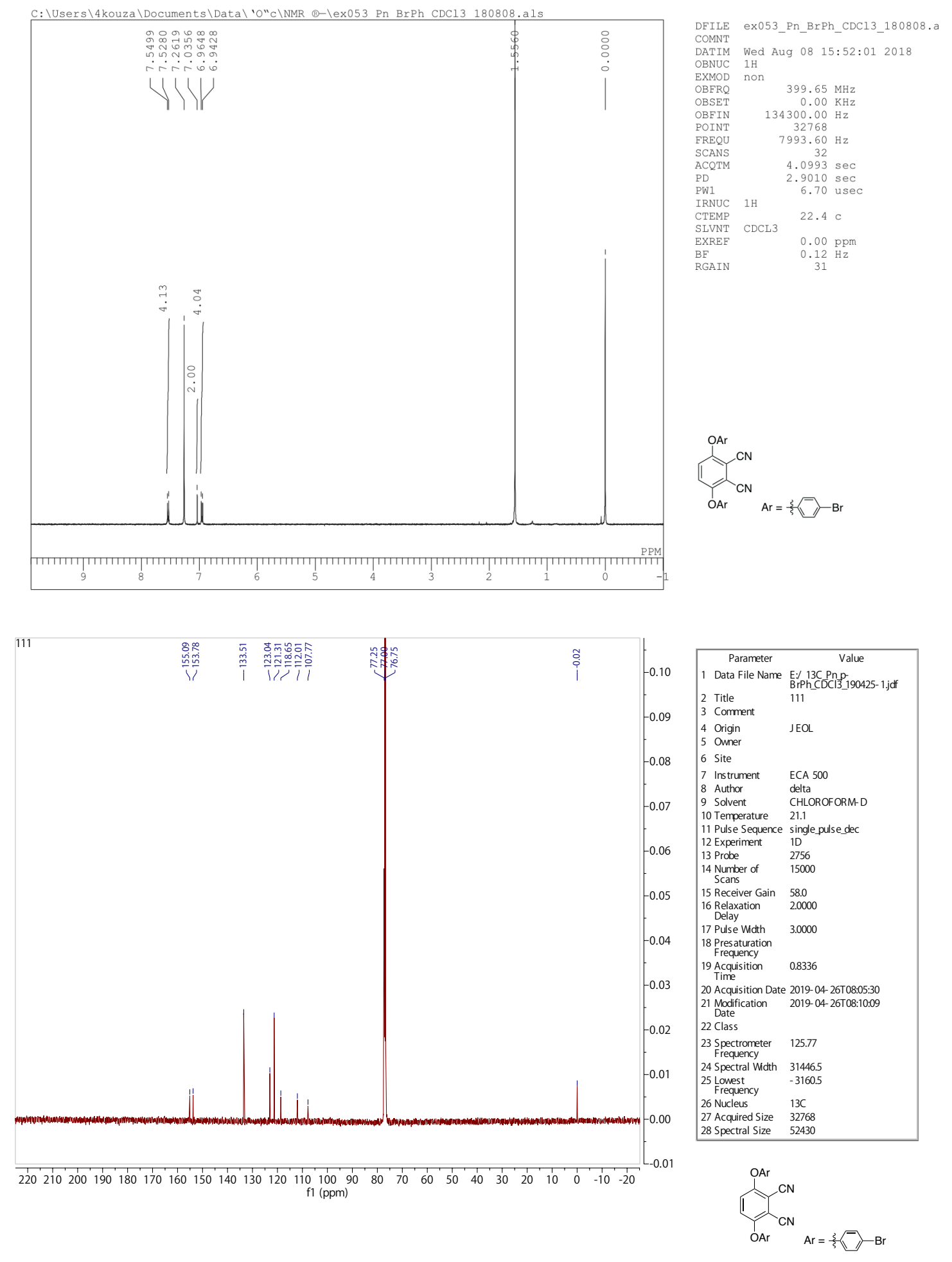

Figure S21. ${ }^{1} \mathrm{H}$ (top) and ${ }^{13} \mathrm{C}$ (bottom) NMR spectra of $\mathbf{1} \mathbf{c}$ in $\mathrm{CDCl}_{3}$. 

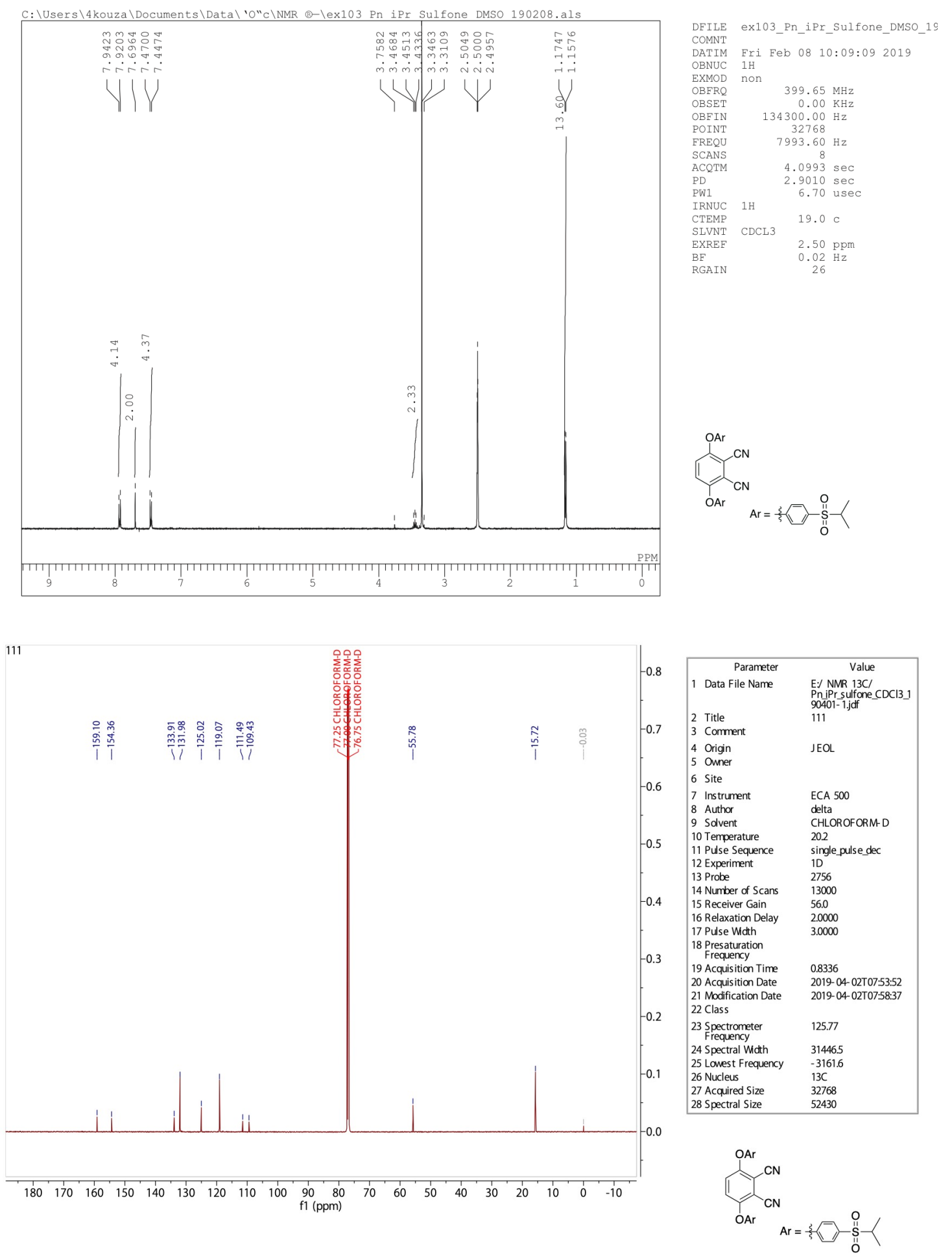

Figure S22. ${ }^{1} \mathrm{H}$ (in DMSO- $d_{6}$ ) (top) and ${ }^{13} \mathrm{C}$ (in $\mathrm{CDCl}_{3}$ ) (bottom) NMR spectra of $\mathbf{1 d}$. 

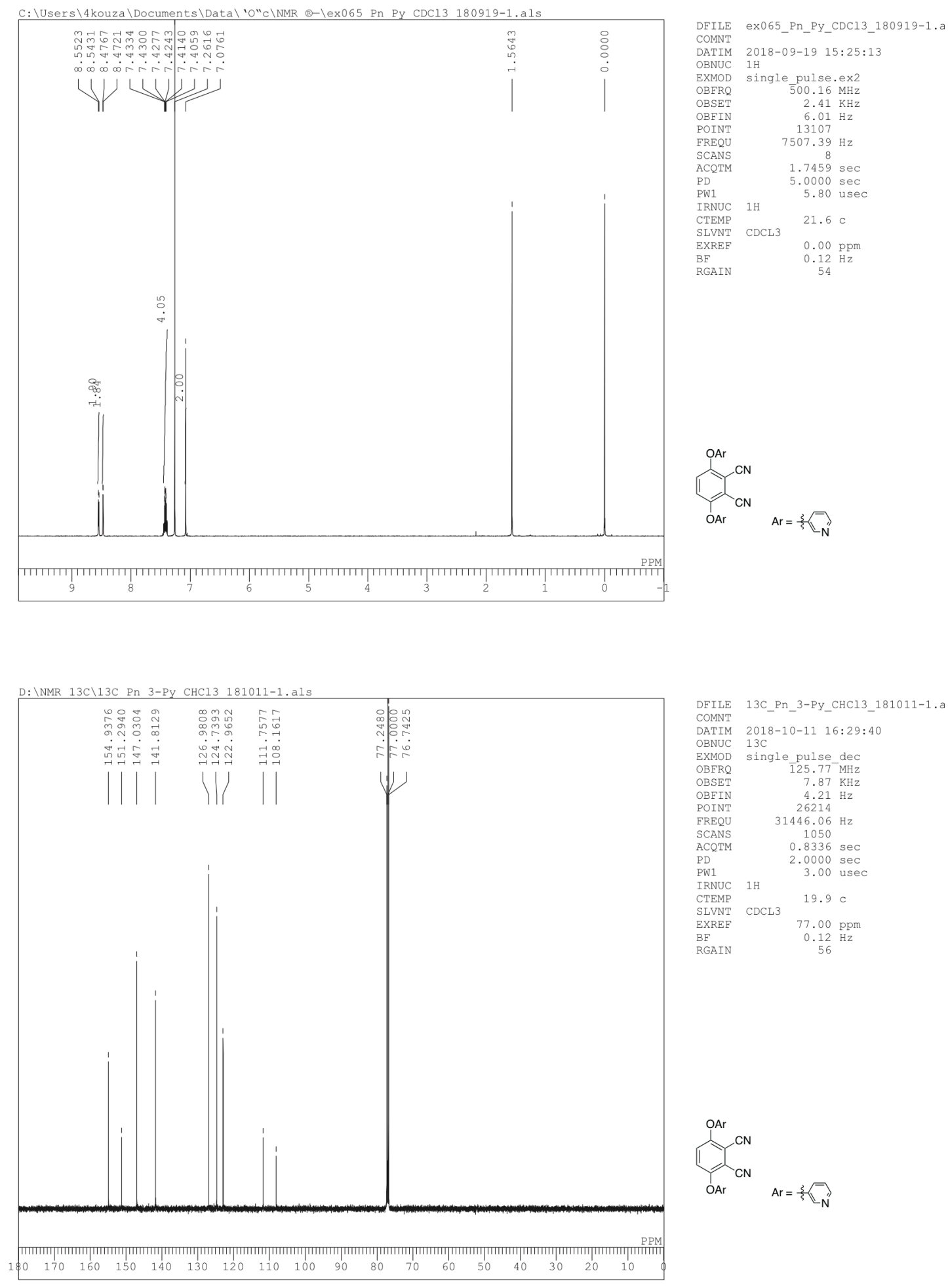

Figure S23. ${ }^{1} \mathrm{H}$ (top) and ${ }^{13} \mathrm{C}$ (bottom) NMR spectra of $\mathbf{1 e}$ in $\mathrm{CDCl}_{3}$. 


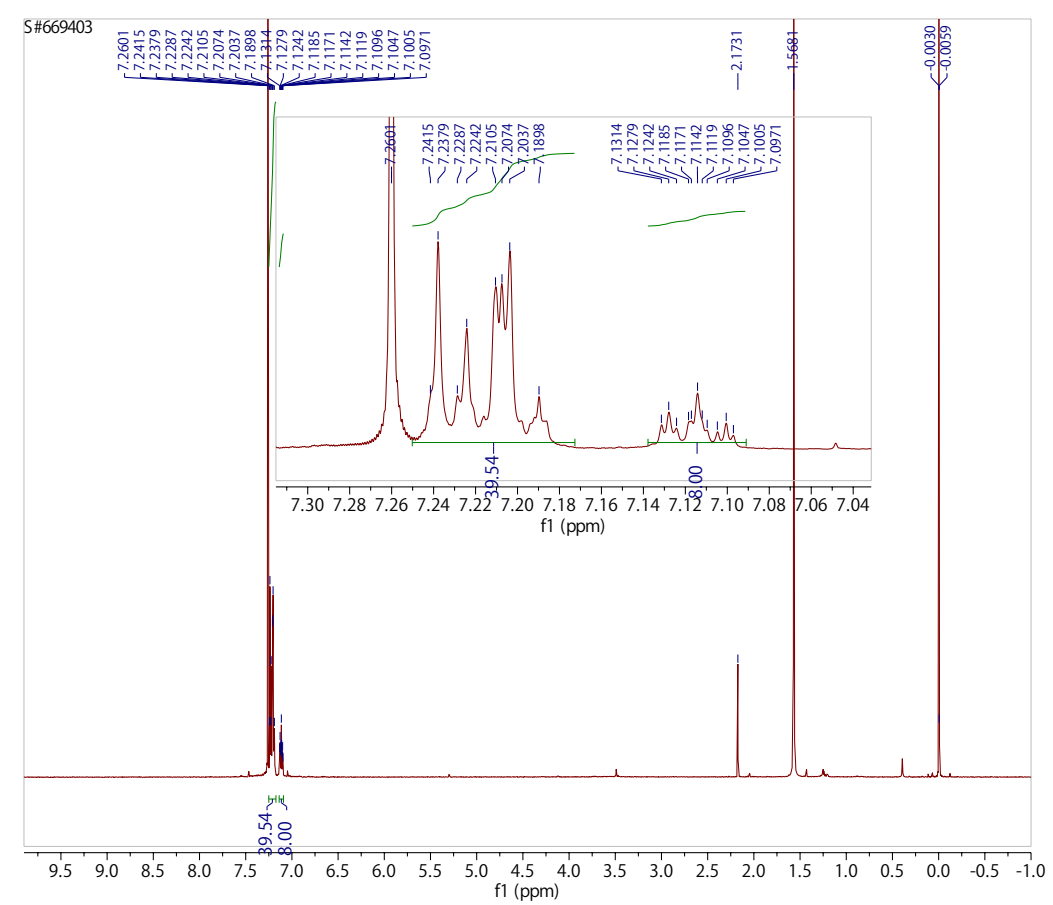

Figure S24. ${ }^{1} \mathrm{H}$ NMR spectrum of $\mathbf{3 a}$ in $\mathrm{CDCl}_{3}$.

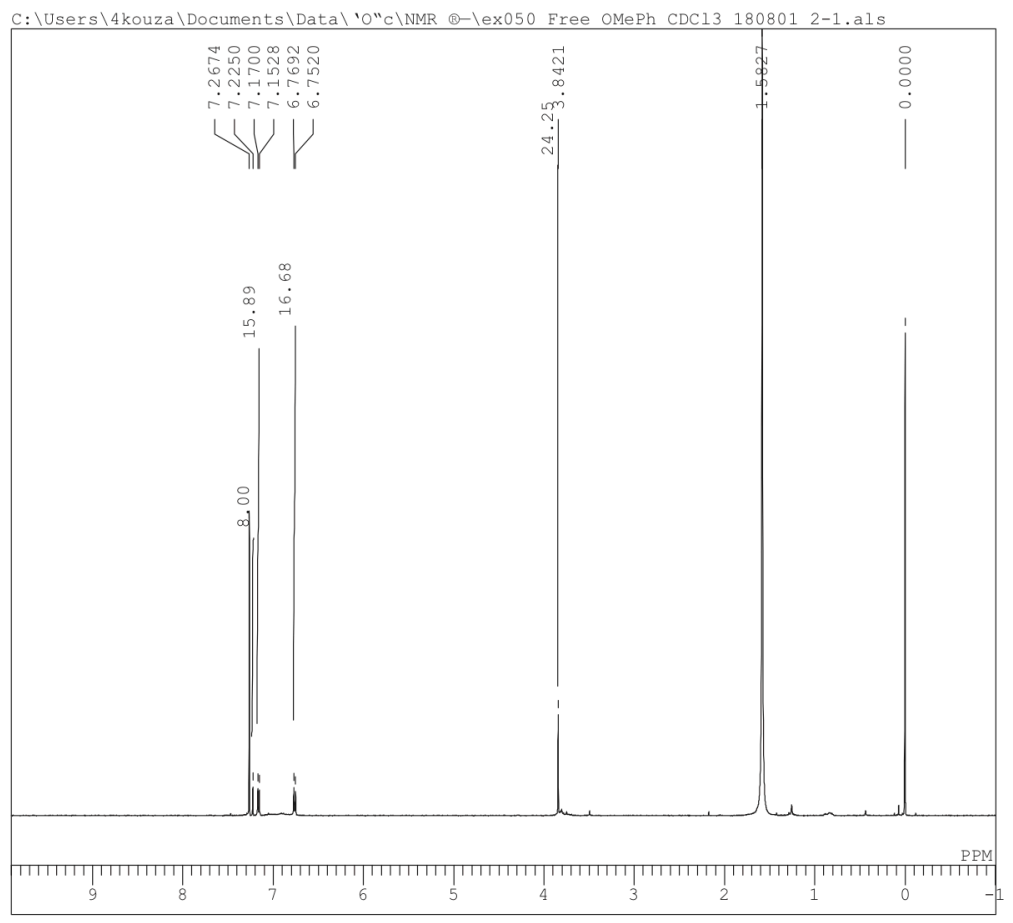

Figure S25. ${ }^{1} \mathrm{H}$ NMR spectrum of $\mathbf{3 b}$ in $\mathrm{CDCl}_{3}$.

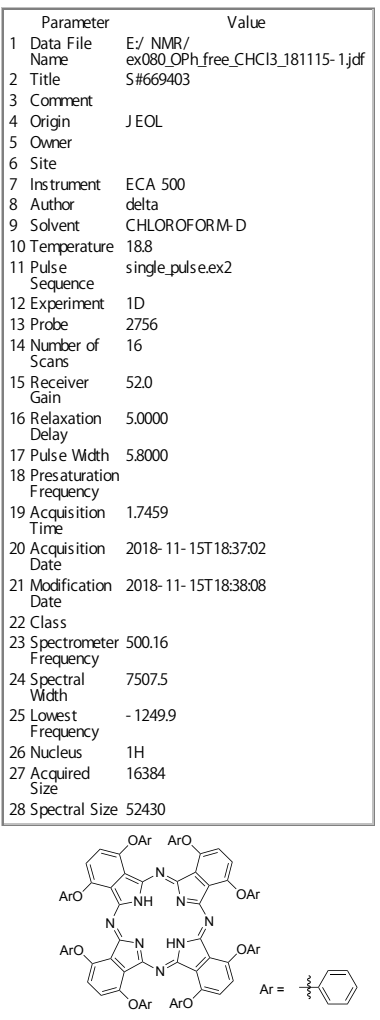

DEILE ex050_Free_OMePh_CDCl3_18080

DATIM 2018-08-01 17:55:29

OBNUC $1 \mathrm{H}$

EXMOD single pulse.ex2
OBFRQ
$500.16 \mathrm{MHz}$

$\begin{array}{rr}\text { OBERQ } & 500.16 \mathrm{MHz} \\ \text { OBSET } & 2.41 \mathrm{KHz}\end{array}$

$\begin{array}{ll}\text { OBSET } & 2.41 \mathrm{KHz} \\ \text { OBEIN } & 6.01 \mathrm{~Hz}\end{array}$

POINT $\quad 13107$

FREQU $\quad 7507.39 \mathrm{~Hz}$

$\begin{array}{lr}\text { SCANS } & 32 \\ \text { ACQTM } & 1.7459 \mathrm{sec}\end{array}$

PD $\quad 5.0000 \mathrm{sec}$

PWI $1 \mathrm{H} \quad 5.80$ usec

CTEMP $21.9 \mathrm{C}$

SLVNT CDCL3 31.90

$\begin{array}{ll}\text { EXREF } & 0.00 \mathrm{ppm} \\ \mathrm{BE} & 0.12 \mathrm{~Hz}\end{array}$

RGAIN

$56 \mathrm{~Hz}$

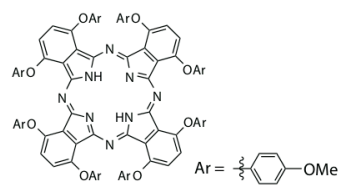



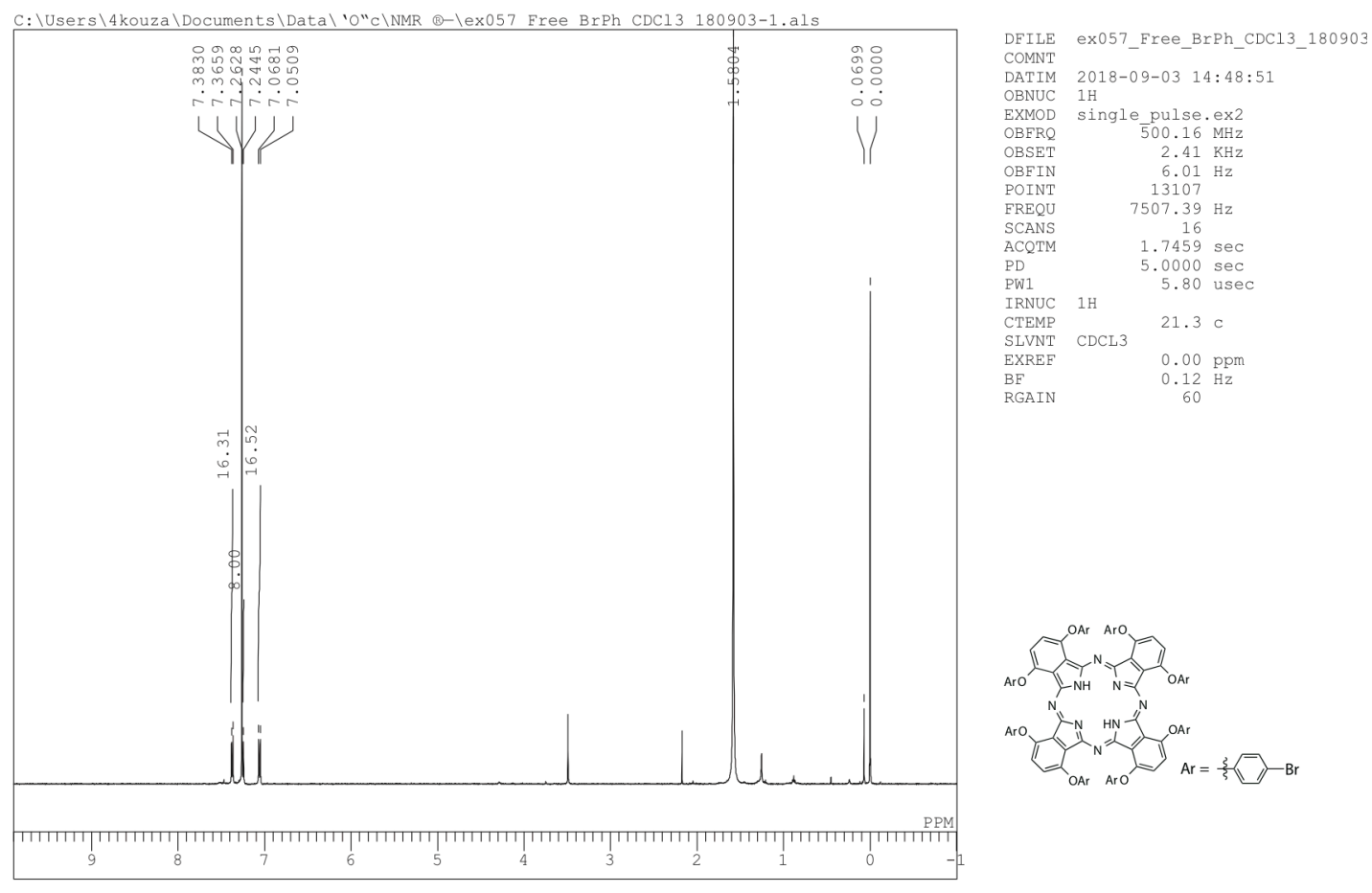

Figure S26. ${ }^{1} \mathrm{H}$ NMR spectrum of $\mathbf{3 c}$ in $\mathrm{CDCl}_{3}$.

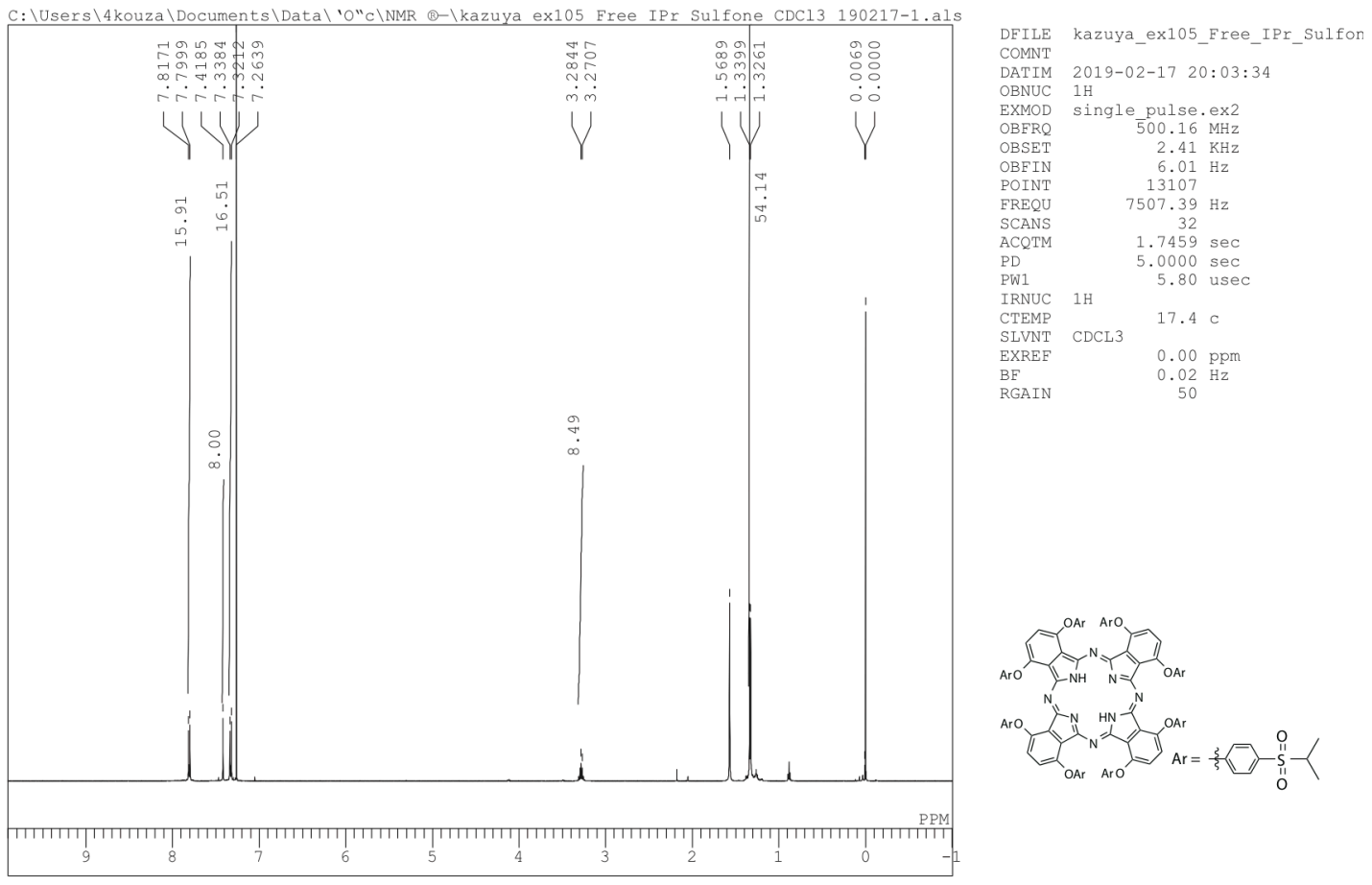

Figure S27. ${ }^{1} \mathrm{H}$ NMR spectrum of $\mathbf{3 d}$ in $\mathrm{CDCl}_{3}$. 

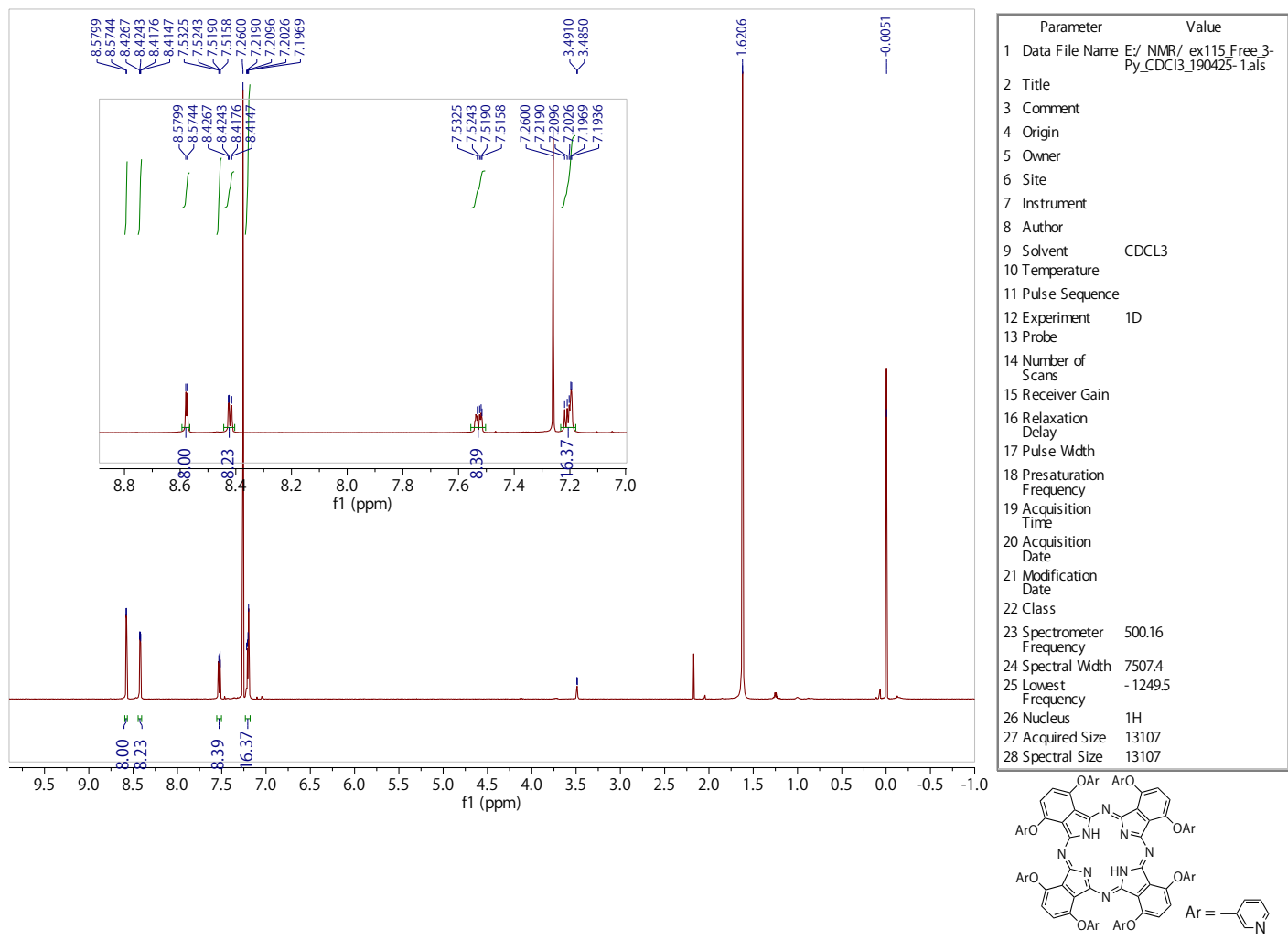

Figure S28. ${ }^{1} \mathrm{H}$ NMR spectrum of $\mathbf{3 e}$ in $\mathrm{CDCl}_{3}$.
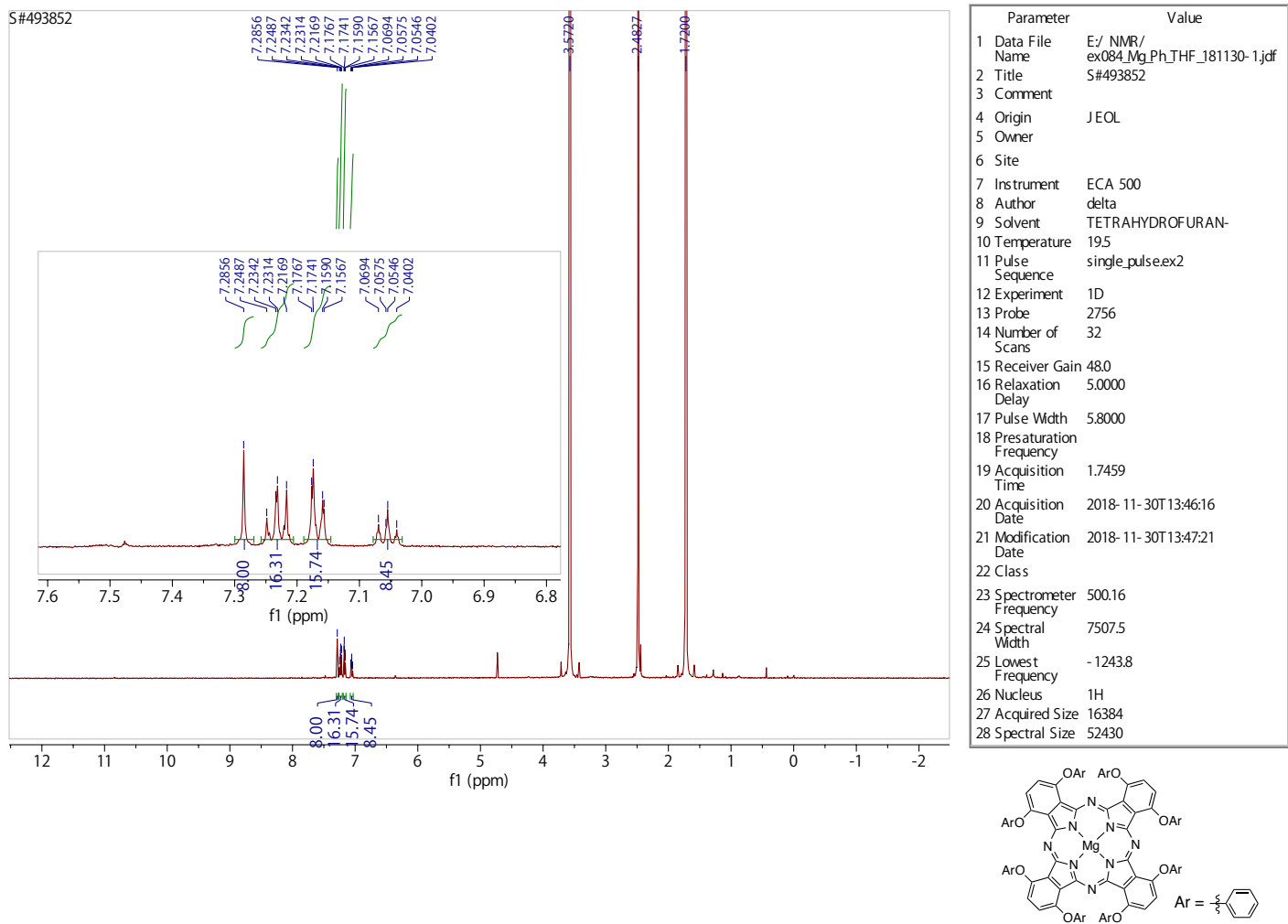

Figure S29. ${ }^{1} \mathrm{H}$ NMR spectrum of 4 a in THF- $d 8$. 

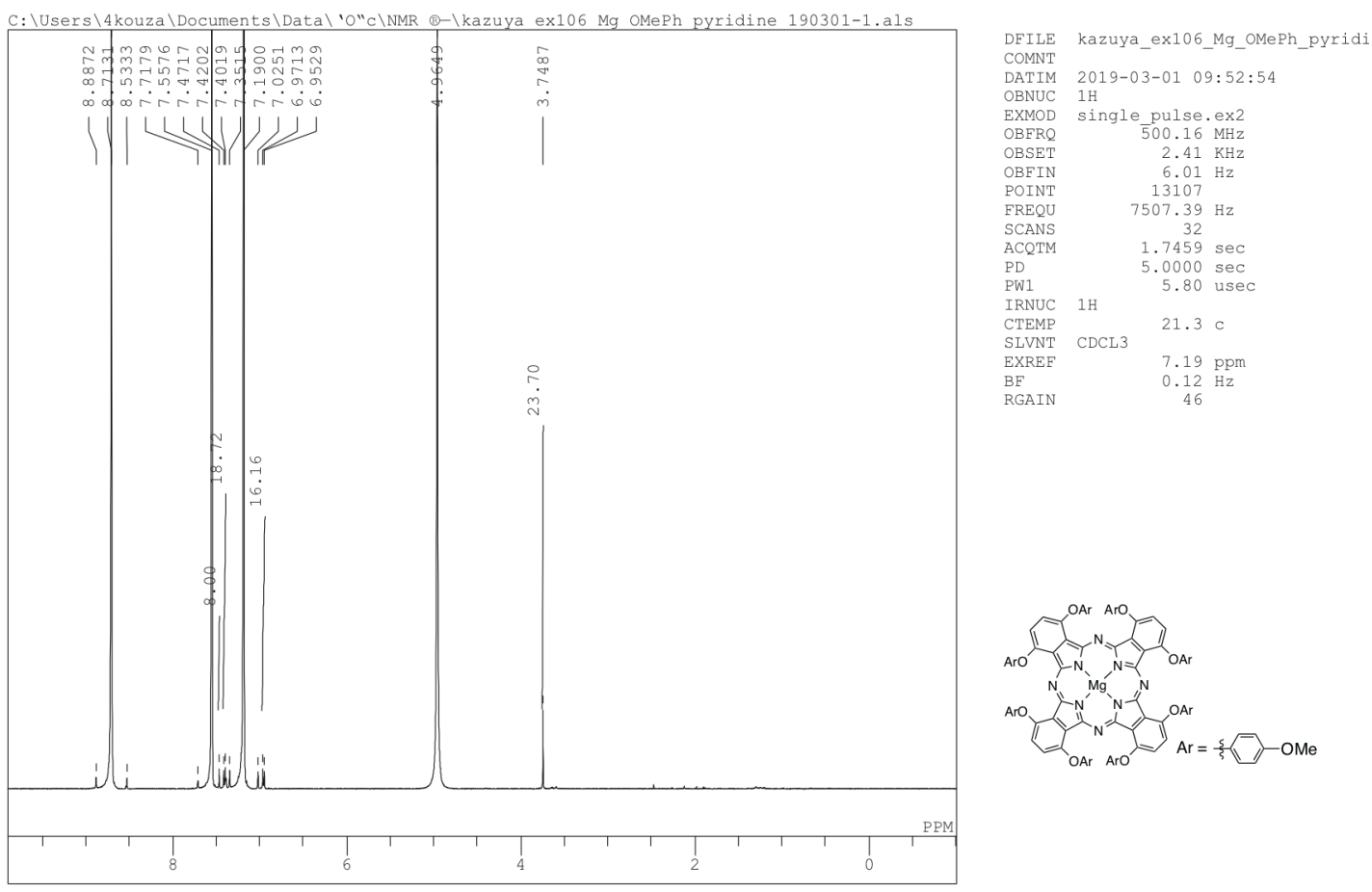

Figure S30. ${ }^{1} \mathrm{H}$ NMR spectrum of $\mathbf{4 b}$ in pyridine- $d 5$.
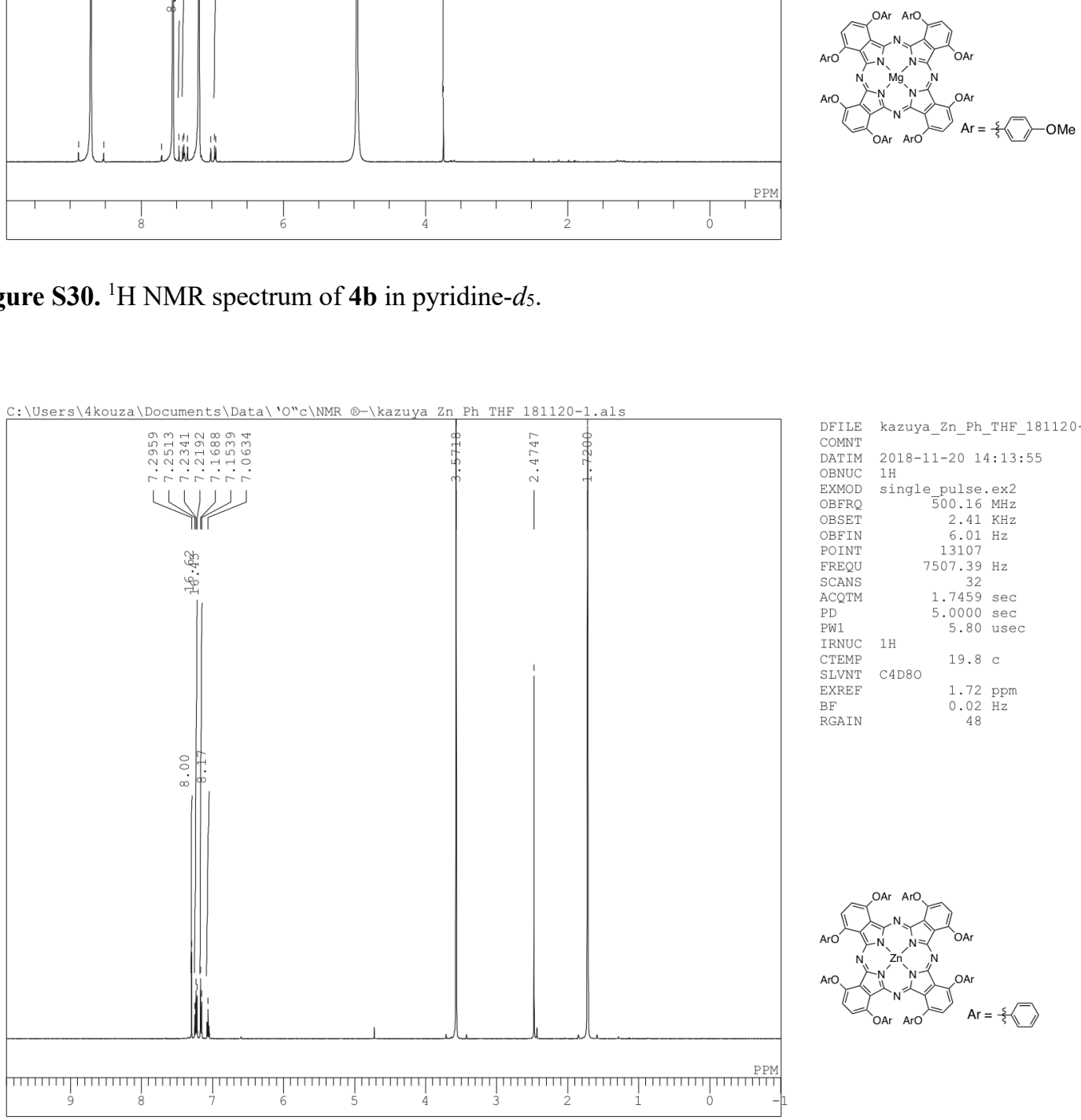

DEILE kazuya_Zn_Ph_THF_181120-1.a1

DATIM 2018-11-20 14:13:55

OBNUC $1 \mathrm{H}$

EXMOD single_pulse.ex2

OBERQ $\quad 500.16 \mathrm{MHz}$

OBEIN $\quad 6.01 \mathrm{~Hz}$

$\begin{array}{lr} & 13107 \\ \text { POINT } & 7507.39\end{array}$

SCANS

$1.7459 \mathrm{se}$

PD $\quad 5.0000 \mathrm{sec}$

IRNUC $1 \mathrm{H}$

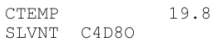

$\begin{array}{lr}\text { ELREF } & 1.72 \mathrm{ppm} \\ \text { BE } & 0.02 \mathrm{~Hz} \\ \text { RGATN } & 48\end{array}$

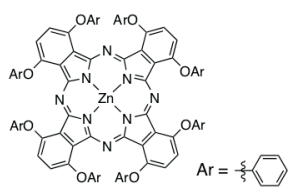

Figure S31. ${ }^{1} \mathrm{H}$ NMR spectrum of $\mathbf{5 a}$ in THF- $d 8$. 

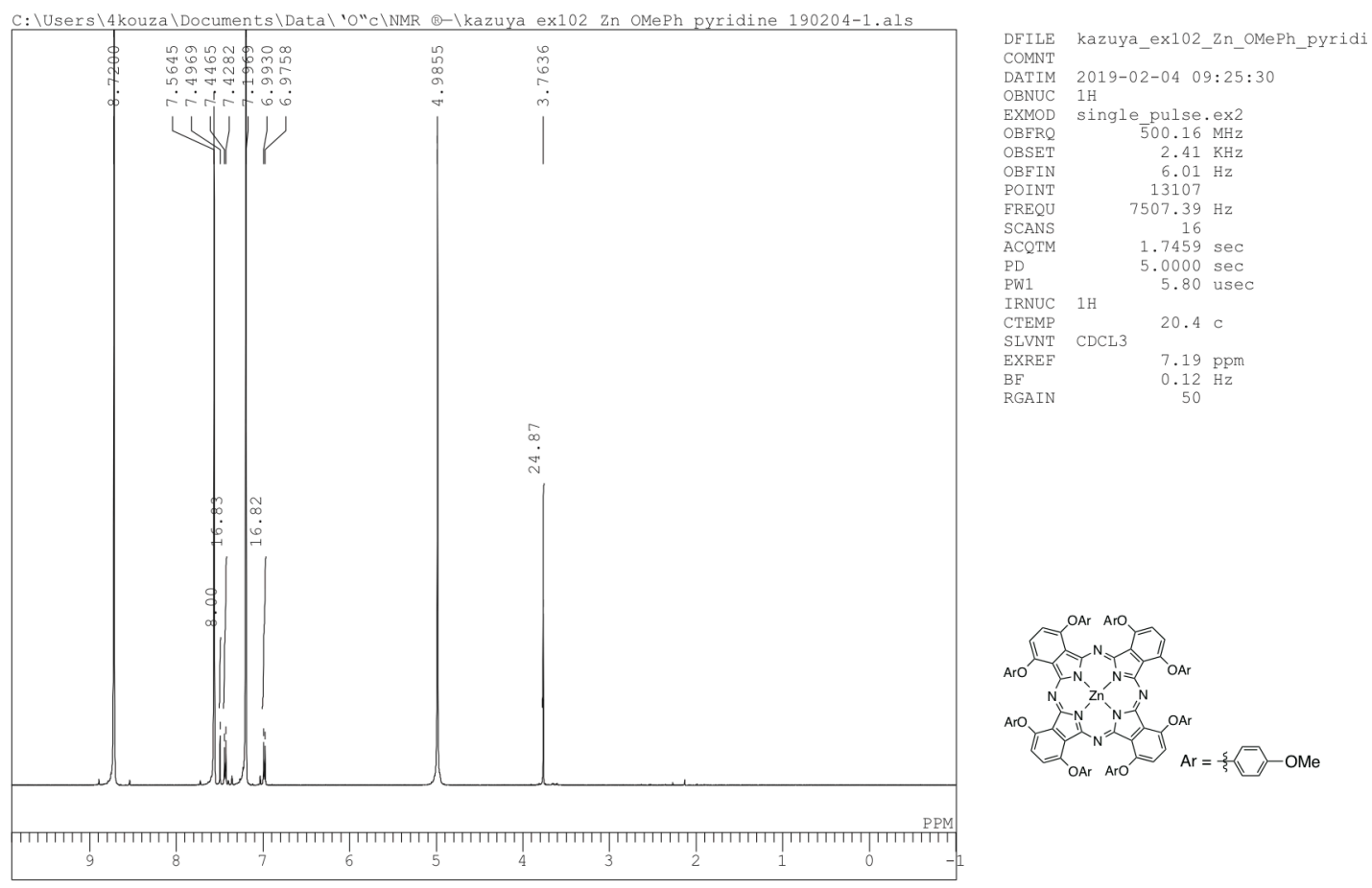

Figure S32. ${ }^{1} \mathrm{H}$ NMR spectrum of $\mathbf{5 b}$ in pyridine- $d 5$.

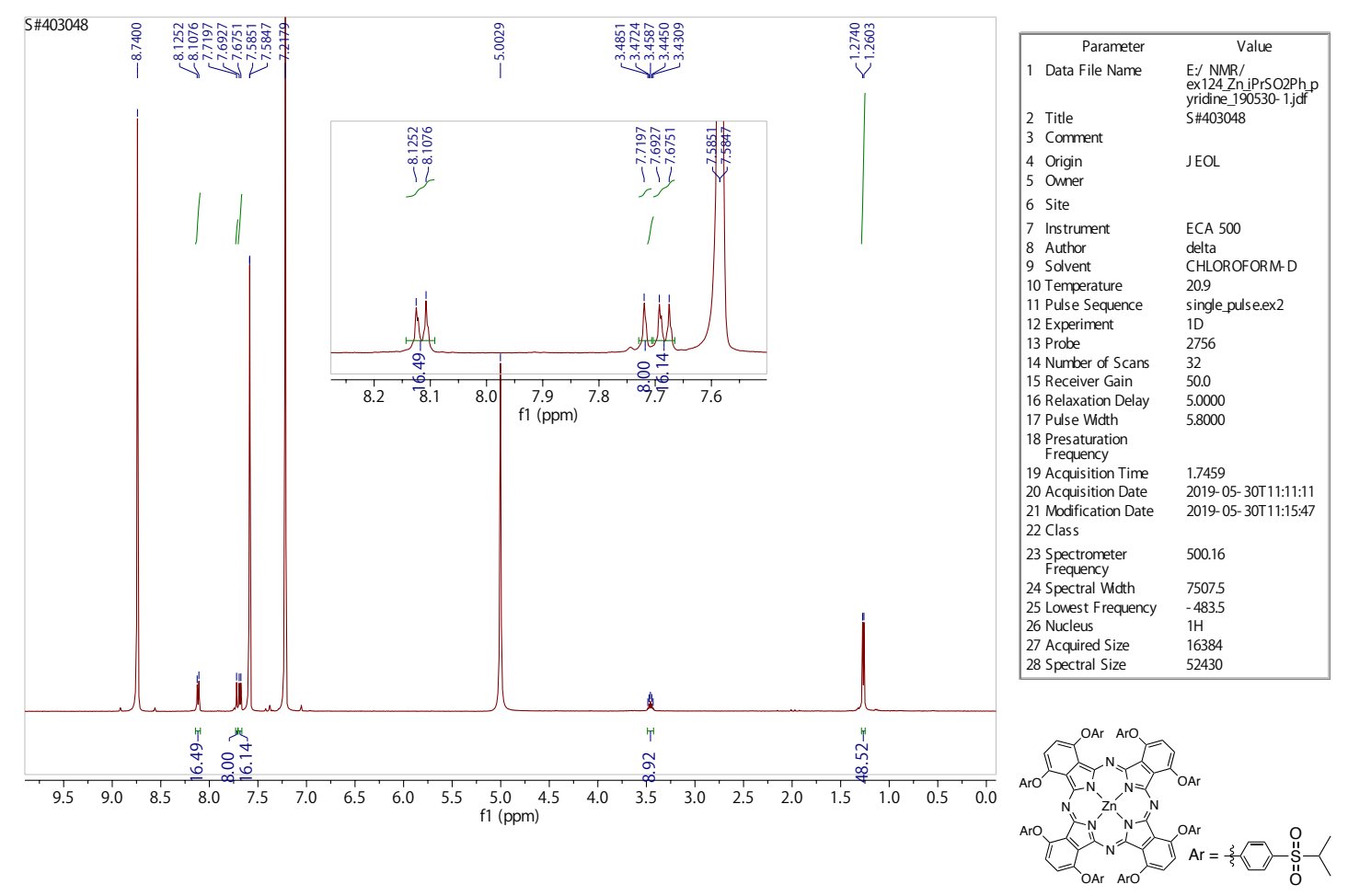

Figure S33. ${ }^{1} \mathrm{H}$ NMR spectrum of $\mathbf{5 d}$ in pyridine- $d 5$. 

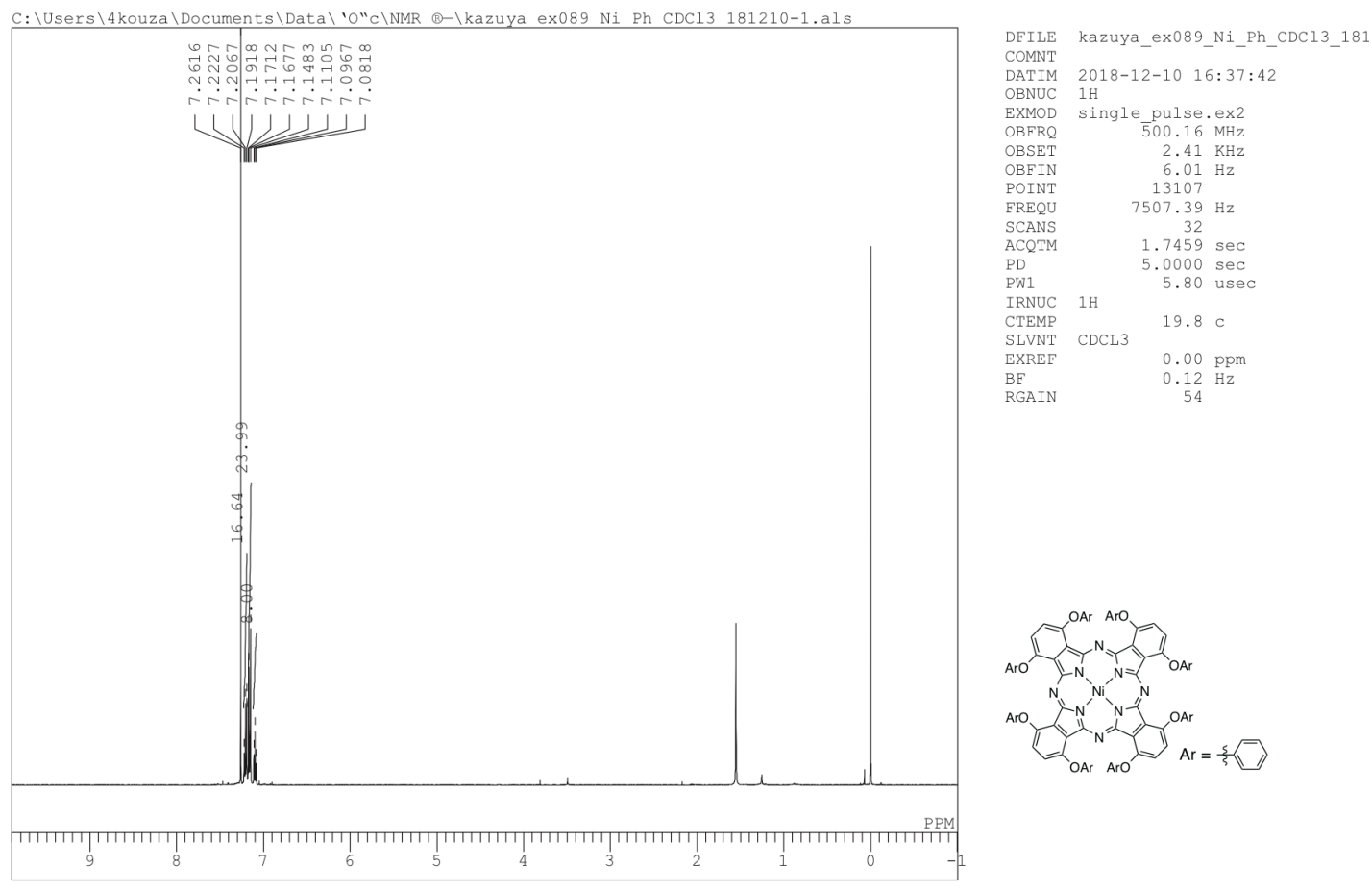

Figure S34. ${ }^{1} \mathrm{H}$ NMR spectrum of $\mathbf{6 a}$ in $\mathrm{CDCl}_{3}$.
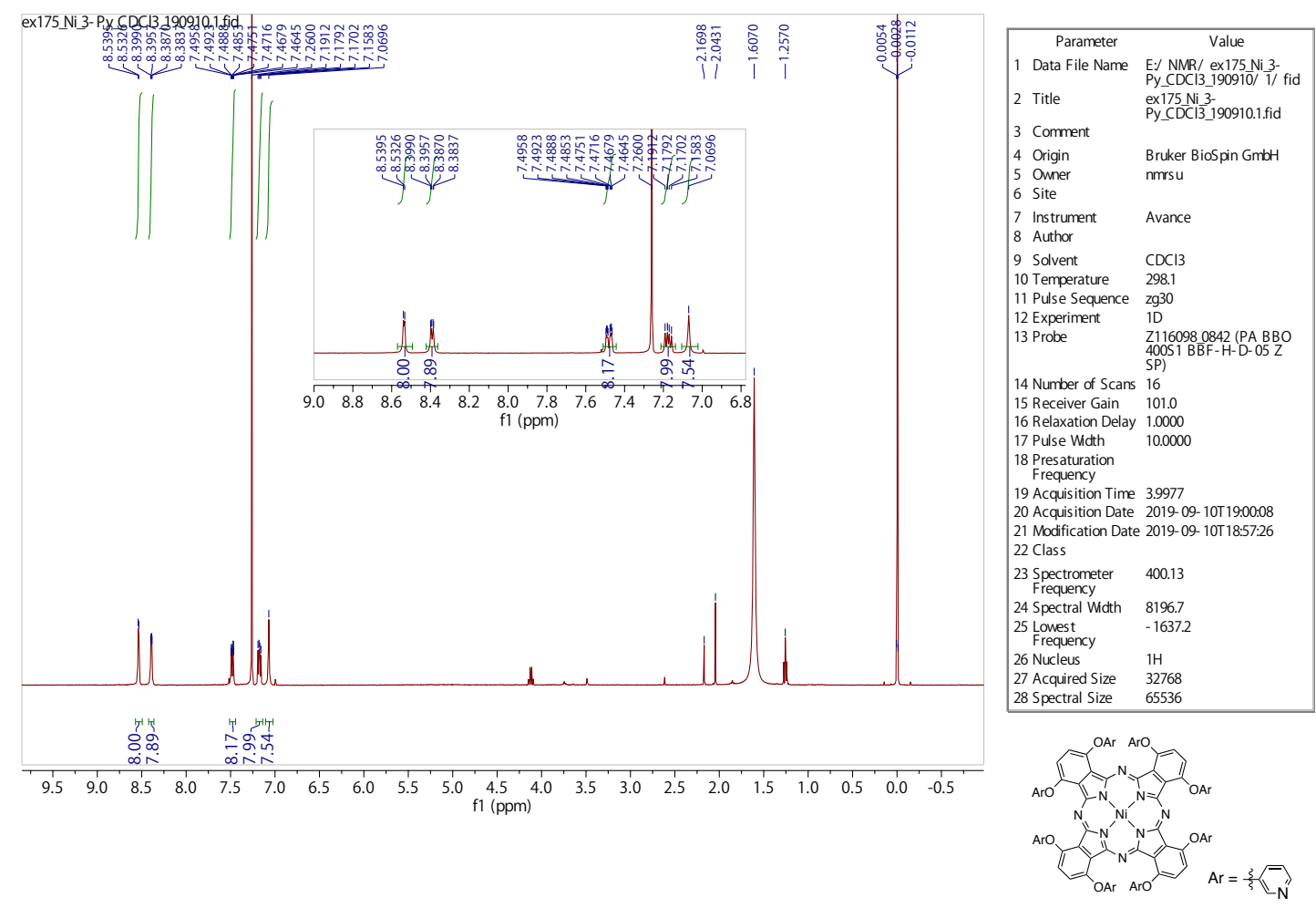

Figure S35. ${ }^{1} \mathrm{H}$ NMR spectrum of $\mathbf{6 e}$ in $\mathrm{CDCl}_{3}$. 

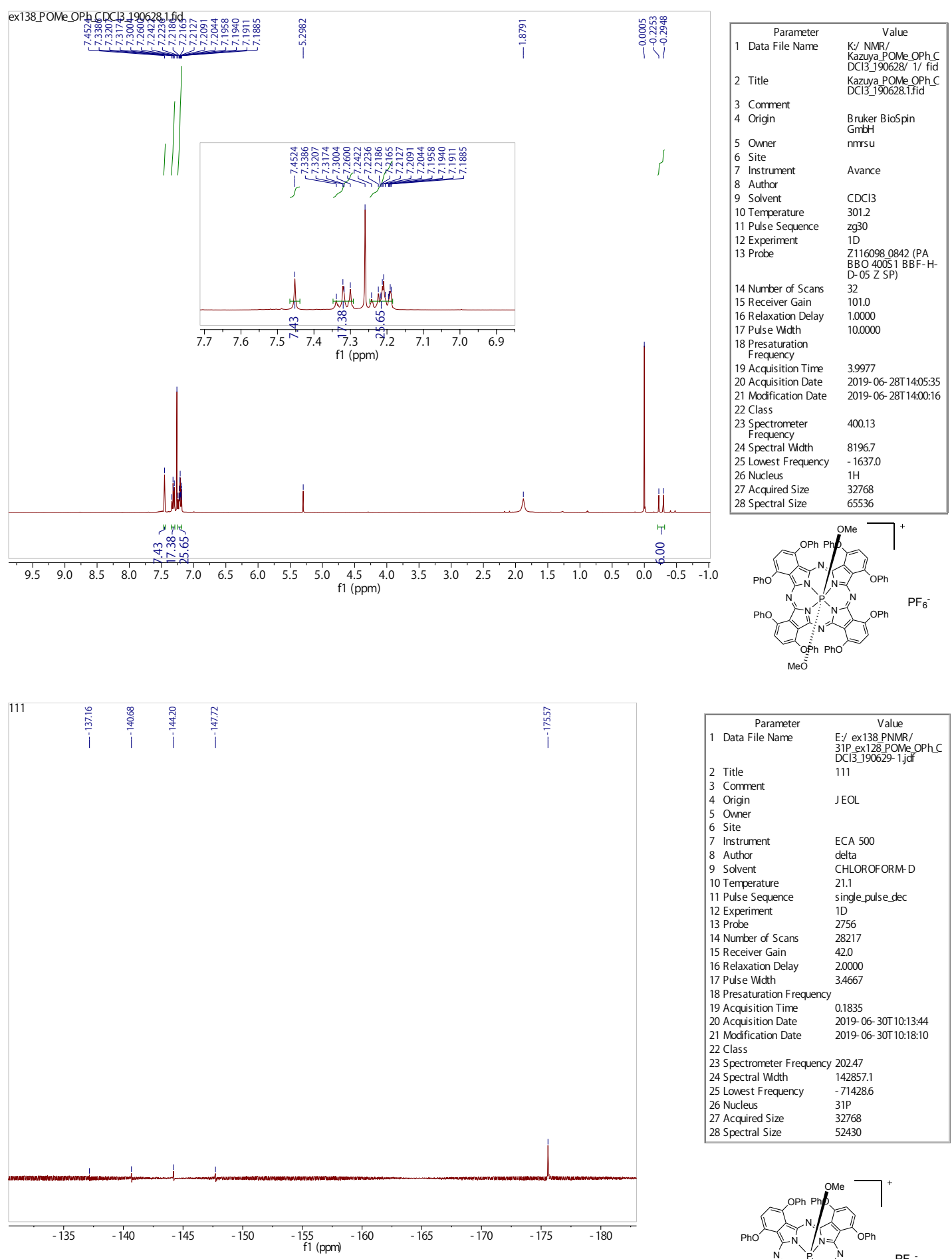

\begin{tabular}{|c|c|}
\hline $\begin{array}{l}\text { Parameter } \\
1 \text { Data File Name }\end{array}$ & $\begin{array}{c}\text { Value } \\
\text { E:/ exx38PNMR/ } \\
\text { 31P ex128POMe_OPh_ } \\
\text { DCI3_190629-1.jff }\end{array}$ \\
\hline 2 Title & \\
\hline 3 Comment & \\
\hline 4 Origin & JEOL \\
\hline 5 Ouner & \\
\hline 6 Site & \\
\hline $\begin{array}{l}7 \text { Instrument } \\
8 \text { Author }\end{array}$ & ECA 500 \\
\hline $\begin{array}{ll}8 & \text { Author } \\
9 & \text { Solvent }\end{array}$ & $\begin{array}{l}\text { delta } \\
\text { CHLOROFORM-D }\end{array}$ \\
\hline 10 Temperature & \\
\hline 11 Pulse Sequence & single_pulse_dec \\
\hline 12 Experiment & 1D \\
\hline 13 Probe & 2756 \\
\hline 14 Number of Scans & 28217 \\
\hline 15 Receiver Gain & 42.0 \\
\hline 16 Relaxation Delay & 2.0000 \\
\hline 17 Pulse Width & 3.4667 \\
\hline 18 Presaturation Frequency & \\
\hline 19 Acquisition Time & 0.1835 \\
\hline 20 Acquisition Date & 2019- 06-30T 10:13:44 \\
\hline 21 Modification Date & 2019- 06-30T 10:18:10 \\
\hline $\begin{array}{l}22 \text { Class } \\
23 \text { Spectrometer Frequency }\end{array}$ & 202.47 \\
\hline 24 Spectral Width & 142857.1 \\
\hline 25 Lowest Frequency & -71428.6 \\
\hline 26 Nucleus & $31 \mathrm{P}$ \\
\hline 27 Acquired Size & 32768 \\
\hline 28 Spectral Size & 5243 \\
\hline
\end{tabular}

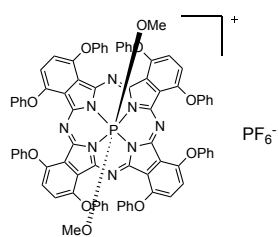

Figure S36. ${ }^{1} \mathrm{H}$ (top) and ${ }^{31} \mathrm{P}$ (bottom) NMR spectra of $\mathbf{8 a}$ in $\mathrm{CDCl}_{3}$. 


\section{Full Computational Details}

\section{Computational Details}

Geometry optimization for all molecules was performed at the DFT level, by means of the hybrid Becke3LYP 6 (B3LYP) functional as implemented in Gaussian 2009. ${ }^{7}$ The 6-31G(d) basis set was used for the all atoms. After the geometry optimization, the time-dependent (TD) DFT calculations ${ }^{8}$ were performed to evaluate the stick absorption spectrum employing the same level and basis set. All stationary points were optimized without any symmetry assumptions and characterized by normal coordinate analysis at the same level of the theory (the number of imaginary frequency, Nimag, 0 ).

\section{Cartesian Coordinates and Total Electron Energies}

$3 a$

\begin{tabular}{|c|c|c|c|c|c|}
\hline \multirow{2}{*}{$\begin{array}{l}\text { Center } \\
\text { Number }\end{array}$} & \multirow{2}{*}{$\begin{array}{l}\text { Atomic } \\
\text { Number }\end{array}$} & \multirow{2}{*}{$\begin{array}{c}\text { Atomic } \\
\text { Type }\end{array}$} & \multicolumn{3}{|c|}{ Coordinates (Angstroms) } \\
\hline & & & $\mathrm{X}$ & $\mathrm{Y}$ & $\mathrm{Z}$ \\
\hline 1 & 6 & 0 & -2.358939 & 3.448845 & 0.463202 \\
\hline 2 & 6 & 0 & -3.393044 & 2.494748 & 0.369332 \\
\hline 3 & 6 & 0 & -2.722682 & 1.211318 & 0.118194 \\
\hline 4 & 7 & 0 & -1.371387 & 1.384756 & 0.031853 \\
\hline 5 & 6 & 0 & -1.119583 & 2.714539 & 0.176170 \\
\hline 6 & 6 & 0 & 3.481979 & 2.387642 & -0.348539 \\
\hline 7 & 6 & 0 & 2.475251 & 3.384523 & -0.425160 \\
\hline 8 & 6 & 0 & 1.196773 & 2.735161 & -0.180204 \\
\hline 9 & 7 & 0 & 1.470832 & 1.391246 & -0.071353 \\
\hline 10 & 6 & 0 & 2.819057 & 1.110389 & -0.136732 \\
\hline 11 & 7 & 0 & 0.043729 & 3.344127 & -0.001365 \\
\hline 12 & 6 & 0 & 2.359054 & -3.448618 & 0.462401 \\
\hline 13 & 6 & 0 & 3.393128 & -2.494516 & 0.368277 \\
\hline 14 & 6 & 0 & 2.722691 & -1.211085 & 0.117369 \\
\hline 15 & 7 & 0 & 1.371382 & -1.384514 & 0.031374 \\
\hline 16 & 6 & 0 & 1.119613 & -2.714307 & 0.175754 \\
\hline 17 & 7 & 0 & 3.398173 & -0.066364 & -0.012380 \\
\hline 18 & 6 & 0 & -3.482110 & -2.387404 & -0.347421 \\
\hline 19 & 6 & 0 & -2.475406 & -3.384275 & -0.424399 \\
\hline 20 & 6 & 0 & -1.196854 & -2.734915 & -0.179886 \\
\hline 21 & 7 & 0 & -1.470873 & -1.391000 & -0.070939 \\
\hline 22 & 6 & 0 & -2.819124 & -1.110158 & -0.135860 \\
\hline 23 & 7 & 0 & -3.398195 & 0.066606 & -0.011338 \\
\hline 24 & 7 & 0 & -0.043748 & -3.343885 & -0.001428 \\
\hline 25 & 6 & 0 & 2.637315 & -4.777615 & 0.789728 \\
\hline 26 & 6 & 0 & 3.974240 & -5.152283 & 0.943935 \\
\hline 27 & 6 & 0 & 5.008278 & -4.221839 & 0.805882 \\
\hline 28 & 6 & 0 & 4.731716 & -2.875120 & 0.527940 \\
\hline 29 & 6 & 0 & 4.839610 & 2.734444 & -0.472419 \\
\hline 30 & 6 & 0 & 5.160613 & 4.077315 & -0.687860 \\
\hline 31 & 6 & 0 & 4.155943 & 5.048538 & -0.809451 \\
\hline 32 & 6 & 0 & 2.808480 & 4.718996 & -0.698160 \\
\hline 33 & 6 & 0 & -4.839797 & -2.734228 & -0.470773 \\
\hline 34 & 6 & 0 & -5.160859 & -4.077094 & -0.686116 \\
\hline 35 & 6 & 0 & -4.156206 & -5.048298 & -0.808127 \\
\hline 36 & 6 & 0 & -2.808719 & -4.718752 & -0.697306 \\
\hline 37 & 6 & 0 & -2.637113 & 4.777823 & 0.790673 \\
\hline
\end{tabular}




\begin{tabular}{|c|c|c|c|c|c|}
\hline 38 & 6 & 0 & -3.973994 & 5.152494 & 0.945295 \\
\hline 39 & 6 & 0 & -5.008064 & 4.222053 & 0.807482 \\
\hline 40 & 6 & 0 & -4.731579 & 2.875356 & 0.529366 \\
\hline 41 & 8 & 0 & 1.850905 & 5.699652 & -0.840376 \\
\hline 42 & 8 & 0 & 5.765580 & 1.732351 & -0.424750 \\
\hline 43 & 8 & 0 & 5.704159 & -1.913000 & 0.476207 \\
\hline 44 & 8 & 0 & 1.647464 & -5.726942 & 0.949356 \\
\hline 45 & 8 & 0 & -1.851166 & -5.699393 & -0.839835 \\
\hline 46 & 8 & 0 & -5.765713 & -1.732124 & -0.422399 \\
\hline 47 & 8 & 0 & -5.704095 & 1.913283 & 0.477920 \\
\hline 48 & 8 & 0 & -1.647214 & 5.727144 & 0.950024 \\
\hline 49 & 6 & 0 & -0.957592 & 5.771020 & 2.152594 \\
\hline 50 & 6 & 0 & 1.175701 & 5.791966 & -2.052015 \\
\hline 51 & 6 & 0 & 7.092397 & 2.018064 & -0.139628 \\
\hline 52 & 6 & 0 & 6.987864 & -2.230721 & 0.069931 \\
\hline 53 & 6 & 0 & 0.958210 & -5.770822 & 2.152139 \\
\hline 54 & 6 & 0 & -1.176388 & -5.791759 & -2.051692 \\
\hline 55 & 6 & 0 & -7.092749 & -2.018261 & -0.138585 \\
\hline 56 & 6 & 0 & -6.987475 & 2.230641 & 0.070392 \\
\hline 57 & 6 & 0 & -0.053446 & 6.455342 & -2.027169 \\
\hline 58 & 6 & 0 & -0.755926 & 6.635528 & -3.216907 \\
\hline 59 & 6 & 0 & -0.247788 & 6.153041 & -4.426550 \\
\hline 60 & 6 & 0 & 0.975975 & 5.484268 & -4.434318 \\
\hline 61 & 6 & 0 & 1.696934 & 5.300919 & -3.251374 \\
\hline 62 & 6 & 0 & 0.249650 & 6.475530 & 2.147143 \\
\hline 63 & 6 & 0 & 0.969903 & 6.608426 & 3.332181 \\
\hline 64 & 6 & 0 & 0.501339 & 6.039628 & 4.520259 \\
\hline 65 & 6 & 0 & -0.700234 & 5.331900 & 4.509383 \\
\hline 66 & 6 & 0 & -1.438261 & 5.193788 & 3.330832 \\
\hline 67 & 6 & 0 & -7.234067 & 2.941760 & -1.108206 \\
\hline 68 & 6 & 0 & -8.552845 & 3.166850 & -1.504028 \\
\hline 69 & 6 & 0 & -9.616964 & 2.679995 & -0.740145 \\
\hline 70 & 6 & 0 & -9.355089 & 1.959257 & 0.427083 \\
\hline 71 & 6 & 0 & -8.040900 & 1.734513 & 0.839171 \\
\hline 72 & 6 & 0 & -7.460685 & -2.600961 & 1.077341 \\
\hline 73 & 6 & 0 & -8.813773 & -2.799060 & 1.353848 \\
\hline 74 & 6 & 0 & -9.789392 & -2.411928 & 0.430727 \\
\hline 75 & 6 & 0 & -9.406224 & -1.820378 & -0.774761 \\
\hline 76 & 6 & 0 & -8.055207 & -1.623567 & -1.066665 \\
\hline 77 & 6 & 0 & 0.052578 & -6.455497 & -2.027296 \\
\hline 78 & 6 & 0 & 0.754669 & -6.635722 & -3.217254 \\
\hline 79 & 6 & 0 & 0.246324 & -6.152927 & -4.426689 \\
\hline 80 & 6 & 0 & -0.977247 & -5.483804 & -4.434013 \\
\hline 81 & 6 & 0 & -1.697817 & -5.300403 & -3.250839 \\
\hline 82 & 6 & 0 & -0.249030 & -6.475333 & 2.147062 \\
\hline 83 & 6 & 0 & -0.968918 & -6.608240 & 3.332324 \\
\hline 84 & 6 & 0 & -0.499991 & -6.039448 & 4.520260 \\
\hline 85 & 6 & 0 & 0.701578 & -5.331718 & 4.509013 \\
\hline 86 & 6 & 0 & 1.439241 & -5.193599 & 3.330236 \\
\hline 87 & 6 & 0 & 7.235274 & -2.941145 & -1.108909 \\
\hline 88 & 6 & 0 & 8.554334 & -3.166679 & -1.503538 \\
\hline 89 & 6 & 0 & 9.617920 & -2.680951 & -0.738192 \\
\hline 90 & 6 & 0 & 9.355244 & -1.960900 & 0.429278 \\
\hline 91 & 6 & 0 & 8.040747 & -1.735717 & 0.840161 \\
\hline 92 & 6 & 0 & 7.459450 & 2.601835 & 1.076060 \\
\hline 93 & 6 & 0 & 8.812378 & 2.799420 & 1.353724 \\
\hline 94 & 6 & 0 & 9.788722 & 2.410720 & 0.432040 \\
\hline 95 & 6 & 0 & 9.406425 & 1.818084 & -0.773194 \\
\hline 96 & 6 & 0 & 8.055600 & 1.621781 & -1.066274 \\
\hline 97 & 1 & 0 & 0.765292 & 0.672513 & 0.044320 \\
\hline 98 & 1 & 0 & -0.765293 & -0.672269 & 0.044487 \\
\hline 99 & 1 & 0 & 4.198920 & -6.184491 & 1.194430 \\
\hline 100 & 1 & 0 & 6.036701 & -4.537594 & 0.941784 \\
\hline 101 & 1 & 0 & 6.199840 & 4.367951 & -0.790324 \\
\hline 102 & 1 & 0 & 4.421424 & 6.081002 & -1.014204 \\
\hline 103 & 1 & 0 & -6.200111 & -4.367775 & -0.788157 \\
\hline 104 & 1 & 0 & -4.421758 & -6.080761 & -1.012796 \\
\hline
\end{tabular}




\begin{tabular}{|c|c|c|c|c|c|}
\hline 105 & 1 & 0 & -4.198598 & 6.184687 & 1.195917 \\
\hline 106 & 1 & 0 & -6.036459 & 4.537781 & 0.943689 \\
\hline 107 & 1 & 0 & -0.456643 & 6.789208 & -1.078425 \\
\hline 108 & 1 & 0 & -1.714136 & 7.148005 & -3.194163 \\
\hline 109 & 1 & 0 & -0.802430 & 6.292896 & -5.350031 \\
\hline 110 & 1 & 0 & 1.383235 & 5.101128 & -5.366425 \\
\hline 111 & 1 & 0 & 2.651728 & 4.786868 & -3.266022 \\
\hline 112 & 1 & 0 & 0.621016 & 6.882610 & 1.213949 \\
\hline 113 & 1 & 0 & 1.909814 & 7.154262 & 3.322944 \\
\hline 114 & 1 & 0 & 1.068787 & 6.144076 & 5.440646 \\
\hline 115 & 1 & 0 & -1.077140 & 4.881938 & 5.424292 \\
\hline 116 & 1 & 0 & -2.374882 & 4.647508 & 3.331868 \\
\hline 117 & 1 & 0 & -6.402976 & 3.306670 & -1.703214 \\
\hline 118 & 1 & 0 & -8.746821 & 3.719992 & -2.419209 \\
\hline 119 & 1 & 0 & -10.641271 & 2.858000 & -1.055100 \\
\hline 120 & 1 & 0 & -10.174745 & 1.568770 & 1.023773 \\
\hline 121 & 1 & 0 & -7.815791 & 1.170340 & 1.738242 \\
\hline 122 & 1 & 0 & -6.694734 & -2.886310 & 1.791675 \\
\hline 123 & 1 & 0 & -9.105170 & -3.251422 & 2.297959 \\
\hline 124 & 1 & 0 & -10.841407 & -2.567107 & 0.652701 \\
\hline 125 & 1 & 0 & -10.158615 & -1.509398 & -1.494087 \\
\hline 126 & 1 & 0 & -7.735642 & -1.156894 & -1.992622 \\
\hline 127 & 1 & 0 & 0.455952 & -6.789611 & -1.078714 \\
\hline 128 & 1 & 0 & 1.712737 & -7.148479 & -3.194852 \\
\hline 129 & 1 & 0 & 0.800663 & -6.292816 & -5.350347 \\
\hline 130 & 1 & 0 & -1.384666 & -5.100419 & -5.365950 \\
\hline 131 & 1 & 0 & -2.652463 & -4.786070 & -3.265148 \\
\hline 132 & 1 & 0 & -0.620696 & -6.882404 & 1.213985 \\
\hline 133 & 1 & 0 & -1.908830 & -7.154079 & 3.323368 \\
\hline 134 & 1 & 0 & -1.067152 & -6.143902 & 5.440823 \\
\hline 135 & 1 & 0 & 1.078767 & -4.881758 & 5.423808 \\
\hline 136 & 1 & 0 & 2.375861 & -4.647318 & 3.330991 \\
\hline 137 & 1 & 0 & 6.404572 & -3.305177 & -1.705003 \\
\hline 138 & 1 & 0 & 8.748964 & -3.719282 & -2.418905 \\
\hline 139 & 1 & 0 & 10.642457 & -2.859296 & -1.052209 \\
\hline 140 & 1 & 0 & 10.174506 & -1.571304 & 1.027091 \\
\hline 141 & 1 & 0 & 7.815012 & -1.172052 & 1.739395 \\
\hline 142 & 1 & 0 & 6.692990 & 2.888421 & 1.789348 \\
\hline 143 & 1 & 0 & 9.103061 & 3.252622 & 2.297654 \\
\hline 144 & 1 & 0 & 10.840600 & 2.565520 & 0.654927 \\
\hline 145 & 1 & 0 & 10.159357 & 1.505857 & -1.491412 \\
\hline 146 & 1 & 0 & 7.736697 & 1.154320 & -1.992061 \\
\hline
\end{tabular}

\section{TD-DFT output}

HOMO: 325, LUMO: 326

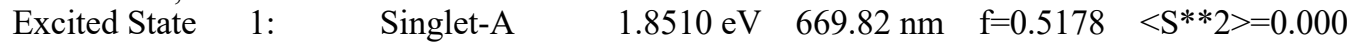

$$
\begin{array}{ll}
325->326 & 0.66003 \\
325->327 & 0.21978
\end{array}
$$

This state for optimization and/or second-order correction.

Total Energy, E(TD-HF/TD-KS) = -4118.24936206

\begin{tabular}{|c|c|c|c|c|c|c|}
\hline $\begin{array}{l}\text { Excited State } \\
316->326 \\
325->326 \\
325->327\end{array}$ & 2: & $\begin{array}{c}\text { Singlet-A } \\
0.12348 \\
-0.22043 \\
0.65156\end{array}$ & $1.8628 \mathrm{eV}$ & $665.59 \mathrm{~nm}$ & $\mathrm{f}=0.4373$ & $<\mathrm{S} * * 2>=0.000$ \\
\hline $\begin{array}{l}\text { Excited State } \\
\begin{array}{r}320->326 \\
324->326\end{array}\end{array}$ & 3: & $\begin{array}{l}\text { Singlet-A } \\
0.11035 \\
0.69398\end{array}$ & $2.5694 \mathrm{eV}$ & $482.55 \mathrm{~nm}$ & $\mathrm{f}=0.0007$ & $<\mathrm{S} * * 2>=0.000$ \\
\hline $\begin{array}{l}\text { Excited State } \\
\quad 323->326\end{array}$ & 4: & $\begin{array}{l}\text { Singlet-A } \\
0.69801\end{array}$ & $2.5808 \mathrm{eV}$ & $480.41 \mathrm{~nm}$ & $\mathrm{f}=0.0078$ & $<\mathrm{S} * * 2>=0.000$ \\
\hline $\begin{array}{l}\text { Excited State } \\
\quad 324->327\end{array}$ & 5: & $\begin{array}{l}\text { Singlet-A } \\
0.69802\end{array}$ & $2.6443 \mathrm{eV}$ & $468.87 \mathrm{~nm}$ & $\mathrm{f}=0.0002$ & $<\mathrm{S} * * 2>=0.000$ \\
\hline
\end{tabular}

Copying the excited state density for this state as the 1-particle RhoCI density. 


\begin{tabular}{|c|c|c|c|c|c|c|}
\hline $\begin{array}{l}\text { Excited State } \\
\begin{array}{r}322->326 \\
323->327\end{array}\end{array}$ & 6: & $\begin{array}{l}\text { Singlet-A } \\
0.69195 \\
0.10806\end{array}$ & $2.6718 \mathrm{eV}$ & $464.05 \mathrm{~nm}$ & $\mathrm{f}=0.0349$ & $<\mathrm{S} * * 2>=0.000$ \\
\hline $\begin{array}{l}\text { Excited State } \\
\quad 321->326\end{array}$ & 7: & $\begin{array}{l}\text { Singlet-A } \\
0.69351\end{array}$ & $2.6754 \mathrm{eV}$ & $463.41 \mathrm{~nm}$ & $\mathrm{f}=0.0003$ & $<\mathrm{S} * * 2>=0.000$ \\
\hline $\begin{array}{l}\text { Excited State } \\
\begin{array}{r}322->326 \\
323->327\end{array}\end{array}$ & 8: & $\begin{array}{c}\text { Singlet-A } \\
-0.11062 \\
0.69436\end{array}$ & $2.6759 \mathrm{eV}$ & $463.34 \mathrm{~nm}$ & $\mathrm{f}=0.0247$ & $<\mathrm{S} * * 2>=0.000$ \\
\hline $\begin{array}{l}\text { Excited State } \\
\qquad 322->327\end{array}$ & 9: & $\begin{array}{l}\text { Singlet-A } \\
0.69800\end{array}$ & $2.7056 \mathrm{eV}$ & $458.25 \mathrm{~nm}$ & $\mathrm{f}=0.0130$ & $<\mathrm{S} * * 2>=0.000$ \\
\hline $\begin{array}{c}\text { Excited State } 1 \\
320->326 \\
321->327\end{array}$ & & $\begin{array}{c}\text { Singlet-A } \\
-0.16330 \\
0.67539\end{array}$ & $2.7160 \mathrm{eV}$ & $456.50 \mathrm{~nm}$ & $\mathrm{f}=0.0010$ & $<\mathrm{S} * * 2>=0.000$ \\
\hline
\end{tabular}

$3 e$

SCF Done: $\mathrm{E}(\mathrm{RB} 3 \mathrm{LYP})=-4246.59413400$ A.U.

\begin{tabular}{|c|c|c|c|c|c|}
\hline \multirow{2}{*}{$\begin{array}{l}\text { Center } \\
\text { Number }\end{array}$} & Atomic & Atomic & \multicolumn{3}{|c|}{ Coordinates (Angstroms) } \\
\hline & Number & Type & $\mathrm{X}$ & $\mathrm{Y}$ & Z \\
\hline 1 & 6 & 0 & 2.299503 & -3.493622 & 0.470947 \\
\hline 2 & 6 & 0 & 3.353702 & -2.562148 & 0.368526 \\
\hline 3 & 6 & 0 & 2.705565 & -1.257806 & 0.156352 \\
\hline 4 & 7 & 0 & 1.350601 & -1.402083 & 0.087490 \\
\hline 5 & 6 & 0 & 1.072627 & -2.728704 & 0.216178 \\
\hline 6 & 6 & 0 & -3.532893 & -2.337980 & -0.266356 \\
\hline 7 & 6 & 0 & -2.534716 & -3.342947 & -0.357662 \\
\hline 8 & 6 & 0 & -1.249204 & -2.706610 & -0.122610 \\
\hline 9 & 7 & 0 & -1.508661 & -1.361430 & -0.009976 \\
\hline 10 & 6 & 0 & -2.855008 & -1.066000 & -0.067571 \\
\hline 11 & 7 & 0 & -0.102997 & -3.333480 & 0.043837 \\
\hline 12 & 6 & 0 & -2.311650 & 3.491841 & 0.463139 \\
\hline 13 & 6 & 0 & -3.366904 & 2.560102 & 0.364620 \\
\hline 14 & 6 & 0 & -2.720469 & 1.255635 & 0.152623 \\
\hline 15 & 7 & 0 & -1.364168 & 1.400971 & 0.084263 \\
\hline 16 & 6 & 0 & -1.085245 & 2.725637 & 0.210783 \\
\hline 17 & 7 & 0 & -3.416542 & 0.121869 & 0.039522 \\
\hline 18 & 6 & 0 & 3.522673 & 2.336647 & -0.250444 \\
\hline 19 & 6 & 0 & 2.526658 & 3.342466 & -0.344971 \\
\hline 20 & 6 & 0 & 1.238113 & 2.706132 & -0.120349 \\
\hline 21 & 7 & 0 & 1.495652 & 1.359050 & -0.010365 \\
\hline 22 & 6 & 0 & 2.841628 & 1.063977 & -0.061254 \\
\hline 23 & 7 & 0 & 3.402798 & -0.123557 & 0.045191 \\
\hline 24 & 7 & 0 & 0.092401 & 3.331587 & 0.041368 \\
\hline 25 & 6 & 0 & -2.565848 & 4.834240 & 0.754577 \\
\hline 26 & 6 & 0 & -3.894124 & 5.251044 & 0.865145 \\
\hline 27 & 6 & 0 & -4.945840 & 4.342899 & 0.714476 \\
\hline 28 & 6 & 0 & -4.694737 & 2.985330 & 0.476529 \\
\hline 29 & 6 & 0 & -4.892956 & -2.681494 & -0.362272 \\
\hline 30 & 6 & 0 & -5.225994 & -4.019871 & -0.583362 \\
\hline 31 & 6 & 0 & -4.230988 & -4.997200 & -0.729353 \\
\hline 32 & 6 & 0 & -2.881831 & -4.673445 & -0.631801 \\
\hline 33 & 6 & 0 & 4.883530 & 2.677690 & -0.332663 \\
\hline 34 & 6 & 0 & 5.219337 & 4.018783 & -0.539686 \\
\hline 35 & 6 & 0 & 4.227383 & 4.996974 & -0.687283 \\
\hline 36 & 6 & 0 & 2.876107 & 4.672987 & -0.608987 \\
\hline 37 & 6 & 0 & 2.553056 & -4.834894 & 0.762868 \\
\hline 38 & 6 & 0 & 3.882364 & -5.253021 & 0.864090 \\
\hline 39 & 6 & 0 & 4.932685 & -4.345685 & 0.709464 \\
\hline 40 & 6 & 0 & 4.681699 & -2.986648 & 0.473742 \\
\hline 41 & 8 & 0 & -1.931189 & -5.661731 & -0.795579 \\
\hline 42 & 8 & 0 & -5.823292 & -1.681734 & -0.290487 \\
\hline
\end{tabular}




\begin{tabular}{|c|c|c|c|c|c|}
\hline 43 & 8 & 0 & -5.717989 & 2.067671 & 0.421691 \\
\hline 44 & 8 & 0 & -1.557022 & 5.763927 & 0.924479 \\
\hline 45 & 8 & 0 & 1.927548 & 5.662073 & -0.771280 \\
\hline 46 & 8 & 0 & 5.809533 & 1.675510 & -0.259403 \\
\hline 47 & 8 & 0 & 5.703518 & -2.069242 & 0.411737 \\
\hline 48 & 8 & 0 & 1.543627 & -5.762707 & 0.932077 \\
\hline 49 & 6 & 0 & 0.847702 & -5.773299 & 2.125808 \\
\hline 50 & 6 & 0 & -1.258276 & -5.726523 & -2.003099 \\
\hline 51 & 6 & 0 & -7.092409 & -1.944744 & 0.191833 \\
\hline 52 & 6 & 0 & -6.837180 & 2.347536 & -0.329493 \\
\hline 53 & 6 & 0 & -0.869410 & 5.775302 & 2.123643 \\
\hline 54 & 6 & 0 & 1.258706 & 5.741027 & -1.979202 \\
\hline 55 & 6 & 0 & 7.087990 & 1.937592 & 0.199660 \\
\hline 56 & 6 & 0 & 6.827952 & -2.357228 & -0.329451 \\
\hline 57 & 6 & 0 & -0.064243 & -6.458954 & -2.022683 \\
\hline 58 & 6 & 0 & 0.211016 & -6.092407 & -4.268381 \\
\hline 59 & 6 & 0 & -0.960064 & -5.341101 & -4.342377 \\
\hline 60 & 6 & 0 & -1.718946 & -5.151527 & -3.186325 \\
\hline 61 & 6 & 0 & -0.374395 & -6.446543 & 2.146468 \\
\hline 62 & 6 & 0 & -1.063217 & -6.516970 & 3.353229 \\
\hline 63 & 6 & 0 & -0.511807 & -5.910077 & 4.484483 \\
\hline 64 & 6 & 0 & 1.322480 & -5.194332 & 3.308280 \\
\hline 65 & 6 & 0 & 6.785500 & -2.974484 & -1.581154 \\
\hline 66 & 6 & 0 & 7.988329 & -3.144620 & -2.264111 \\
\hline 67 & 6 & 0 & 9.170346 & -2.688233 & -1.677560 \\
\hline 68 & 6 & 0 & 8.060833 & -1.935646 & 0.179881 \\
\hline 69 & 6 & 0 & 7.322561 & 2.572690 & 1.424917 \\
\hline 70 & 6 & 0 & 9.586871 & 2.299027 & 1.204953 \\
\hline 71 & 6 & 0 & 9.447261 & 1.641084 & -0.017826 \\
\hline 72 & 6 & 0 & 8.166951 & 1.459715 & -0.538958 \\
\hline 73 & 6 & 0 & 0.061927 & 6.457903 & -2.001507 \\
\hline 74 & 6 & 0 & -0.595004 & 6.595931 & -3.220165 \\
\hline 75 & 6 & 0 & -0.039414 & 6.009811 & -4.360230 \\
\hline 76 & 6 & 0 & 1.739769 & 5.186827 & -3.170703 \\
\hline 77 & 6 & 0 & 0.330354 & 6.498254 & 2.154727 \\
\hline 78 & 6 & 0 & 0.639329 & 6.039533 & 4.378499 \\
\hline 79 & 6 & 0 & -0.537253 & 5.295694 & 4.441002 \\
\hline 80 & 6 & 0 & -1.316264 & 5.157874 & 3.290929 \\
\hline 81 & 6 & 0 & -6.787139 & 2.951071 & -1.587615 \\
\hline 82 & 6 & 0 & -7.985639 & 3.114580 & -2.279752 \\
\hline 83 & 6 & 0 & -9.171680 & 2.665877 & -1.695354 \\
\hline 84 & 6 & 0 & -8.073742 & 1.932715 & 0.176790 \\
\hline 85 & 6 & 0 & -7.305629 & -2.590886 & 1.415346 \\
\hline 86 & 6 & 0 & -9.573200 & -2.311223 & 1.241231 \\
\hline 87 & 6 & 0 & -9.455177 & -1.641652 & 0.022493 \\
\hline 88 & 6 & 0 & -8.184626 & -1.457949 & -0.521262 \\
\hline 89 & 1 & 0 & -0.798125 & -0.646827 & 0.103590 \\
\hline 90 & 1 & 0 & 0.784816 & 0.643679 & 0.095204 \\
\hline 91 & 1 & 0 & -4.096994 & 6.293779 & 1.088308 \\
\hline 92 & 1 & 0 & -5.971643 & 4.682745 & 0.812785 \\
\hline 93 & 1 & 0 & -6.269251 & -4.303162 & -0.667183 \\
\hline 94 & 1 & 0 & -4.506078 & -6.026387 & -0.937196 \\
\hline 95 & 1 & 0 & 6.263234 & 4.302621 & -0.611186 \\
\hline 96 & 1 & 0 & 4.504717 & 6.027978 & -0.881785 \\
\hline 97 & 1 & 0 & 4.085617 & -6.296456 & 1.082708 \\
\hline 98 & 1 & 0 & 5.958569 & -4.686709 & 0.801571 \\
\hline 99 & 1 & 0 & 0.317424 & -6.886239 & -1.099802 \\
\hline 100 & 1 & 0 & 0.821306 & -6.258307 & -5.153989 \\
\hline 101 & 1 & 0 & -1.280793 & -4.911276 & -5.286947 \\
\hline 102 & 1 & 0 & -2.639017 & -4.577537 & -3.208857 \\
\hline 103 & 1 & 0 & -0.770283 & -6.875218 & 1.233138 \\
\hline 104 & 1 & 0 & -2.018812 & -7.029768 & 3.414361 \\
\hline 105 & 1 & 0 & -1.027376 & -5.947978 & 5.442242 \\
\hline 106 & 1 & 0 & 2.270420 & -4.662623 & 3.323029 \\
\hline 107 & 1 & 0 & 5.842158 & -3.304528 & -2.004299 \\
\hline 108 & 1 & 0 & 8.006143 & -3.618213 & -3.241320 \\
\hline 109 & 1 & 0 & 10.123555 & -2.808255 & -2.188874 \\
\hline
\end{tabular}




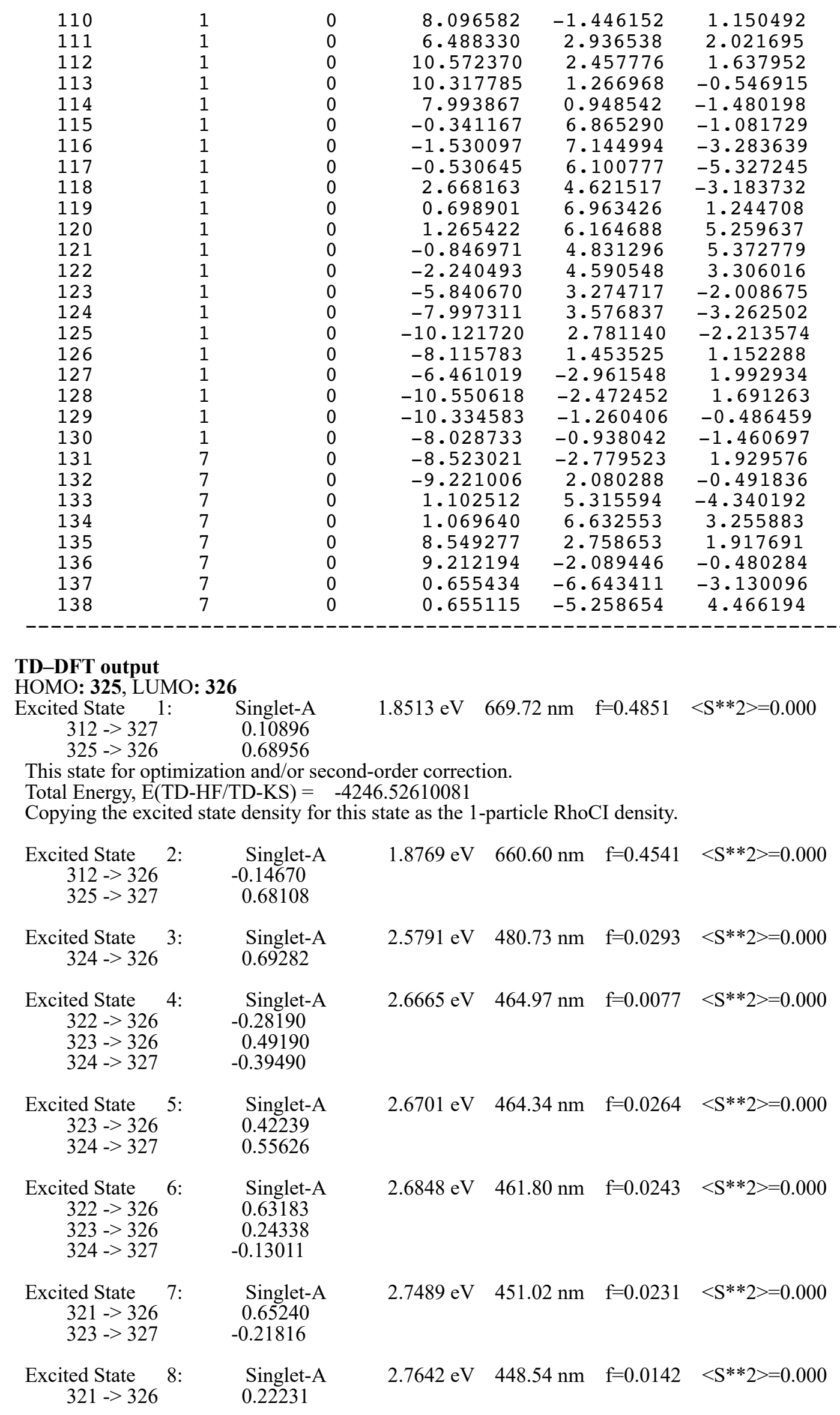




\begin{tabular}{|c|c|c|c|c|c|}
\hline $\begin{array}{l}322->327 \\
323->327\end{array}$ & $\begin{array}{l}0.19058 \\
0.60360\end{array}$ & & & & \\
\hline $\begin{array}{c}\text { Excited State } \\
322->327 \\
323->327\end{array}$ & $\begin{array}{l}\text { Singlet-A } \\
0.67283 \\
-0.18064\end{array}$ & $2.7773 \mathrm{eV}$ & $446.41 \mathrm{~nm}$ & $\mathrm{f}=0.0233$ & $<\mathrm{S} * * 2>=0.000$ \\
\hline $\begin{array}{c}\text { Excited State 10: } \\
320->326 \\
321->327\end{array}$ & $\begin{array}{c}\text { Singlet-A } \\
-0.35842 \\
0.58506\end{array}$ & $2.8312 \mathrm{eV}$ & $437.92 \mathrm{~nm}$ & $\mathrm{f}=0.0102$ & $<\mathrm{S}^{* * 2}>=0.000$ \\
\hline
\end{tabular}

8a'

SCF Done: E (RB3LYP $)=-4688.56327072$ A.U.

\begin{tabular}{|c|c|c|c|c|c|}
\hline \multirow{2}{*}{$\begin{array}{l}\text { Center } \\
\text { Number }\end{array}$} & Atomic & Atomic & \multicolumn{2}{|c|}{ Coordinates } & igstroms ) \\
\hline & Number & 'уре & $\mathrm{X}$ & $\mathrm{Y}$ & $\mathrm{Z}$ \\
\hline 1 & 7 & 0 & -1.205962 & 1.422672 & 0.026217 \\
\hline 2 & 6 & 0 & -2.563416 & 1.338328 & -0.286085 \\
\hline 3 & 6 & 0 & -3.142445 & 2.654984 & -0.260914 \\
\hline 4 & 6 & 0 & -4.447253 & 3.106917 & -0.521841 \\
\hline 5 & 6 & 0 & -4.687982 & 4.477386 & -0.367781 \\
\hline 6 & 6 & 0 & -3.674400 & 5.362944 & 0.017040 \\
\hline 7 & 6 & 0 & -2.367812 & 4.923880 & 0.260386 \\
\hline 8 & 6 & 0 & -2.119366 & 3.545719 & 0.123313 \\
\hline 9 & 6 & 0 & -0.912988 & 2.762647 & 0.258074 \\
\hline 10 & 7 & 0 & 0.280603 & 3.292034 & 0.457109 \\
\hline 11 & 1 & 0 & -5.682362 & 4.866281 & -0.552254 \\
\hline 12 & 1 & 0 & -3.909951 & 6.415474 & 0.125073 \\
\hline 13 & 7 & 0 & 1.455287 & 1.215977 & 0.008864 \\
\hline 14 & 6 & 0 & 1.375330 & 2.577610 & 0.286428 \\
\hline 15 & 6 & 0 & 2.700883 & 3.153709 & 0.234314 \\
\hline 16 & 6 & 0 & 3.170520 & 4.458800 & 0.466317 \\
\hline 17 & 6 & 0 & 4.539076 & 4.687639 & 0.265820 \\
\hline 18 & 6 & 0 & 5.403198 & 3.664068 & -0.134031 \\
\hline 19 & 6 & 0 & 4.946508 & 2.355373 & -0.343070 \\
\hline 20 & 6 & 0 & 3.575680 & 2.121783 & -0.158327 \\
\hline 21 & 6 & 0 & 2.785683 & 0.919620 & -0.257686 \\
\hline 22 & 7 & 0 & 3.313775 & -0.258612 & -0.447688 \\
\hline 23 & 1 & 0 & 4.938834 & 5.683343 & 0.419681 \\
\hline 24 & 1 & 0 & 6.451616 & 3.891210 & -0.287441 \\
\hline 25 & 7 & 0 & 1.249368 & -1.444674 & -0.025962 \\
\hline 26 & 6 & 0 & 2.613097 & -1.348296 & -0.271913 \\
\hline 27 & 6 & 0 & 3.213866 & -2.651337 & -0.168923 \\
\hline 28 & 6 & 0 & 4.533190 & -3.085913 & -0.377471 \\
\hline 29 & 6 & 0 & 4.805238 & -4.437813 & -0.135916 \\
\hline 30 & 6 & 0 & 3.806208 & -5.317752 & 0.288061 \\
\hline 31 & 6 & 0 & 2.483981 & -4.899089 & 0.476328 \\
\hline 32 & 6 & 0 & 2.198171 & -3.542253 & 0.239548 \\
\hline 33 & 6 & 0 & 0.967311 & -2.777094 & 0.289982 \\
\hline 34 & 7 & 0 & -0.233129 & -3.311259 & 0.470606 \\
\hline 35 & 1 & 0 & 5.813749 & -4.809997 & -0.270649 \\
\hline 36 & 1 & 0 & 4.063232 & -6.353744 & 0.480634 \\
\hline 37 & 7 & 0 & -1.417042 & -1.233627 & 0.005043 \\
\hline 38 & 6 & 0 & -1.340074 & -2.603313 & 0.274782 \\
\hline 39 & 6 & 0 & -2.666731 & -3.174818 & 0.187841 \\
\hline 40 & 6 & 0 & -3.157854 & -4.480502 & 0.398398 \\
\hline 41 & 6 & 0 & -4.524450 & -4.691504 & 0.191951 \\
\hline 42 & 6 & 0 & -5.379656 & -3.654915 & -0.196302 \\
\hline 43 & 6 & 0 & -4.909853 & -2.354014 & -0.393577 \\
\hline 44 & 6 & 0 & -3.536319 & -2.131536 & -0.201579 \\
\hline 45 & 6 & 0 & -2.747887 & -0.933295 & -0.281181 \\
\hline 46 & 7 & 0 & -3.264724 & 0.253867 & -0.471069 \\
\hline 47 & 1 & 0 & -4.933943 & -5.685522 & 0.332924 \\
\hline 48 & 1 & 0 & -6.430801 & -3.868922 & -0.351788 \\
\hline 49 & 15 & 0 & 0.026113 & -0.011011 & 0.002451 \\
\hline 50 & 8 & 0 & 0.136373 & -0.080726 & 1.671490 \\
\hline
\end{tabular}




\begin{tabular}{|c|c|c|c|c|c|}
\hline 51 & 8 & 0 & 0.049337 & 0.108656 & -1.667040 \\
\hline 52 & 6 & 0 & -0.194958 & -0.945933 & -2.595586 \\
\hline 53 & 1 & 0 & 0.021272 & -0.521596 & -3.578512 \\
\hline 54 & 1 & 0 & 0.466525 & -1.802859 & -2.426760 \\
\hline 55 & 1 & 0 & -1.237459 & -1.278592 & -2.573551 \\
\hline 56 & 6 & 0 & -0.916549 & 0.174430 & 2.598727 \\
\hline 57 & 1 & 0 & -1.813345 & -0.414333 & 2.375969 \\
\hline 58 & 1 & 0 & -0.527053 & -0.132496 & 3.572033 \\
\hline 59 & 1 & 0 & -1.177375 & 1.236774 & 2.637413 \\
\hline 60 & 8 & 0 & -5.686794 & -1.312264 & -0.800749 \\
\hline 61 & 8 & 0 & -2.295338 & -5.461620 & 0.759021 \\
\hline 62 & 8 & 0 & 1.510441 & -5.719145 & 0.951876 \\
\hline 63 & 8 & 0 & 5.456024 & -2.174940 & -0.786610 \\
\hline 64 & 8 & 0 & 5.731321 & 1.322880 & -0.756853 \\
\hline 65 & 8 & 0 & 2.286530 & 5.411727 & 0.857996 \\
\hline 66 & 8 & 0 & -1.347333 & 5.736013 & 0.641003 \\
\hline 67 & 8 & 0 & -5.382383 & 2.193822 & -0.901445 \\
\hline 68 & 6 & 0 & -6.644806 & 2.617689 & -1.320167 \\
\hline 69 & 6 & 0 & -6.814520 & 3.131490 & -2.606201 \\
\hline 70 & 6 & 0 & -7.727078 & 2.433369 & -0.462677 \\
\hline 71 & 6 & 0 & -8.098254 & 3.474267 & -3.034018 \\
\hline 72 & 1 & 0 & -5.953034 & 3.250224 & -3.256162 \\
\hline 73 & 6 & 0 & -9.007358 & 2.774045 & -0.904814 \\
\hline 74 & 1 & 0 & -7.561200 & 2.009967 & 0.522589 \\
\hline 75 & 6 & 0 & -9.194879 & 3.297167 & -2.185927 \\
\hline 76 & 1 & 0 & -8.240840 & 3.873845 & -4.034045 \\
\hline 77 & 1 & 0 & -9.857554 & 2.628783 & -0.244667 \\
\hline 78 & 1 & 0 & -10.192202 & 3.561185 & -2.525136 \\
\hline 79 & 6 & 0 & -1.504266 & 7.118545 & 0.566062 \\
\hline 80 & 6 & 0 & -1.457904 & 7.758861 & -0.67344 \\
\hline 81 & 6 & 0 & -1.628464 & 7.840028 & 1.751257 \\
\hline 82 & 6 & 0 & -1.545562 & 9.151338 & -0.720034 \\
\hline 83 & 1 & 0 & -1.354180 & 7.169736 & -1.579625 \\
\hline 84 & 6 & 0 & -1.707400 & 9.233317 & 1.691996 \\
\hline 85 & 1 & 0 & -1.644164 & 7.310367 & 2.698340 \\
\hline 86 & 6 & 0 & -1.670576 & 9.890093 & 0.459908 \\
\hline 87 & 1 & 0 & -1.512265 & 9.658484 & -1.680257 \\
\hline 88 & 1 & 0 & -1.798885 & 9.803761 & 2.611762 \\
\hline 89 & 1 & 0 & -1.737774 & 10.973260 & 0.418559 \\
\hline 90 & 6 & 0 & -7.068408 & -1.365150 & -0.625712 \\
\hline 91 & 6 & 0 & -7.875730 & -1.352899 & -1.761250 \\
\hline 92 & 6 & 0 & -7.620016 & -1.353356 & 0.656937 \\
\hline 93 & 6 & 0 & -9.263673 & -1.324873 & -1.607521 \\
\hline 94 & 1 & 0 & -7.412676 & -1.349184 & -2.74273 \\
\hline 95 & 6 & 0 & -9.008313 & -1.333982 & 0.79687 \\
\hline 96 & 1 & 0 & -6.967529 & -1.356526 & 1.52471. \\
\hline 97 & 6 & 0 & -9.832039 & -1.319110 & -0.33216 \\
\hline 98 & 1 & 0 & -9.899249 & -1.310211 & -2.48821 \\
\hline 99 & 1 & 0 & -9.445940 & -1.326825 & 1.791244 \\
\hline 100 & 1 & 0 & -10.911830 & -1.303664 & -0.21734 \\
\hline 101 & 6 & 0 & -2.811335 & -6.660096 & 1.25001 \\
\hline 102 & 6 & 0 & -2.666948 & -7.808807 & 0.47662 \\
\hline 103 & 6 & 0 & -3.385811 & -6.706035 & 2.52127 \\
\hline 104 & 6 & 0 & -3.112691 & -9.029488 & 0.98738 \\
\hline 105 & 1 & 0 & -2.197942 & -7.739496 & -0.49895 \\
\hline 106 & 6 & 0 & -3.831842 & -7.931319 & 3.01834 \\
\hline 107 & 1 & 0 & -3.471949 & -5.796108 & 3.10727 \\
\hline 108 & 6 & 0 & -3.697576 & -9.093428 & 2.25386 \\
\hline 109 & 1 & 0 & -3.001036 & -9.930342 & 0.39137 \\
\hline 110 & 1 & 0 & -4.278243 & -7.977525 & 4.00767 \\
\hline 111 & 1 & 0 & -4.044350 & -10.044947 & 2.64618 \\
\hline 112 & 6 & 0 & 1.575835 & -7.079949 & 0.67991 \\
\hline 113 & 6 & 0 & 1.449036 & -7.954839 & 1.75708 \\
\hline 114 & 6 & 0 & 1.694357 & -7.555024 & -0.62852 \\
\hline 115 & 6 & 0 & 1.439379 & -9.329920 & 1.51940 \\
\hline 116 & 1 & 0 & 1.349221 & -7.550245 & 2.75890 \\
\hline 117 & 6 & 0 & 1.691779 & -8.932998 & -0.85147 \\
\hline
\end{tabular}




\begin{tabular}{|c|c|c|c|c|c|}
\hline & & & & & \\
\hline 118 & 1 & 0 & 1.783001 & -6.855594 & -1.454121 \\
\hline 119 & 6 & 0 & 1.565320 & -9.823217 & 0.218590 \\
\hline 120 & 1 & 0 & 1.338802 & -10.015463 & 2.355768 \\
\hline 121 & 1 & 0 & 1.786071 & -9.309738 & -1.866261 \\
\hline 122 & 1 & 0 & 1.564791 & -10.894269 & 0.038695 \\
\hline 123 & 6 & 0 & 6.724833 & -2.594668 & -1.190689 \\
\hline 124 & 6 & 0 & 7.810147 & -2.309559 & -0.365571 \\
\hline 125 & 6 & 0 & 6.895938 & -3.206255 & -2.433217 \\
\hline 126 & 6 & 0 & 9.094979 & -2.650174 & -0.794472 \\
\hline 127 & 1 & 0 & 7.641521 & -1.815878 & 0.585879 \\
\hline 128 & 6 & 0 & 8.184352 & -3.548075 & -2.847310 \\
\hline 129 & 1 & 0 & 6.032302 & -3.401019 & -3.061675 \\
\hline 130 & 6 & 0 & 9.284222 & -3.271871 & -2.030482 \\
\hline 131 & 1 & 0 & 9.947341 & -2.427193 & -0.159314 \\
\hline 132 & 1 & 0 & 8.327872 & -4.024480 & -3.812967 \\
\hline 133 & 1 & 0 & 10.284984 & -3.536051 & -2.359231 \\
\hline 134 & 6 & 0 & 7.119525 & 1.411330 & -0.674328 \\
\hline 135 & 6 & 0 & 7.755544 & 1.483222 & 0.566803 \\
\hline 136 & 6 & 0 & 7.849628 & 1.343649 & -1.859068 \\
\hline 137 & 6 & 0 & 9.150390 & 1.498136 & 0.612943 \\
\hline 138 & 1 & 0 & 7.163276 & 1.524118 & 1.475843 \\
\hline 139 & 6 & 0 & 9.244884 & 1.350075 & -1.798416 \\
\hline 140 & 1 & 0 & 7.322062 & 1.273769 & -2.804814 \\
\hline 141 & 6 & 0 & 9.897225 & 1.432346 & -0.566532 \\
\hline 142 & 1 & 0 & 9.653093 & 1.556740 & 1.574274 \\
\hline 143 & 1 & 0 & 9.820346 & 1.293593 & -2.717904 \\
\hline 144 & 1 & 0 & 10.982338 & 1.443549 & -0.524575 \\
\hline 145 & 6 & 0 & 2.751260 & 6.649841 & 1.301613 \\
\hline 146 & 6 & 0 & 3.365367 & 6.761640 & 2.549997 \\
\hline 147 & 6 & 0 & 2.499832 & 7.770249 & 0.513414 \\
\hline 148 & 6 & 0 & 3.744246 & 8.024807 & 3.007089 \\
\hline 149 & 1 & 0 & 3.532209 & 5.872652 & 3.150563 \\
\hline 150 & 6 & 0 & 2.876619 & 9.029330 & 0.985622 \\
\hline 151 & 1 & 0 & 1.998214 & 7.648851 & -0.440588 \\
\hline 152 & 6 & 0 & 3.501719 & 9.159059 & 2.227597 \\
\hline 153 & 1 & 0 & 4.221796 & 8.121902 & 3.977990 \\
\hline 154 & 1 & 0 & 2.678612 & 9.908532 & 0.379562 \\
\hline 155 & 1 & 0 & 3.794215 & 10.140144 & 2.590043 \\
\hline
\end{tabular}

\section{TD-DFT output}

\section{HOMO: 348, LUMO: 349}

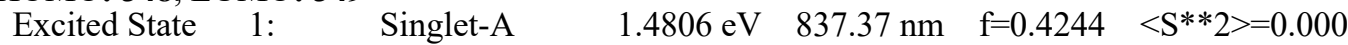
$348->349$ 0.69342

This state for optimization and/or second-order correction.

Total Energy, E(TD-HF/TD-KS) $=-4688.50885843$

Copying the excited state density for this state as the 1-particle RhoCI density.

\begin{tabular}{|c|c|c|c|c|c|c|}
\hline $\begin{array}{l}\text { Excited State } \\
\qquad 348->350\end{array}$ & 2: & $\begin{array}{l}\text { Singlet-A } \\
0.69307\end{array}$ & $1.4879 \mathrm{eV}$ & $833.29 \mathrm{~nm}$ & $\mathrm{f}=0.4184$ & $<\mathrm{S} * * 2>=0.000$ \\
\hline $\begin{array}{l}\text { Excited State } \\
345->349 \\
346->350 \\
347->349\end{array}$ & 3: & $\begin{array}{l}\text { Singlet-A } \\
0.29971 \\
0.24730 \\
0.57579\end{array}$ & $2.0887 \mathrm{eV}$ & $593.59 \mathrm{~nm}$ & $\mathrm{f}=0.1271$ & $<\mathrm{S} * * 2>=0.000$ \\
\hline $\begin{array}{l}\text { Excited State } \\
346->349 \\
346->350 \\
347->350\end{array}$ & 4: & $\begin{array}{l}\text { Singlet-A } \\
0.15533 \\
0.14694 \\
0.66163\end{array}$ & $2.1089 \mathrm{eV}$ & $587.92 \mathrm{~nm}$ & $\mathrm{f}=0.1902$ & $<\mathrm{S}^{* * 2}>=0.000$ \\
\hline $\begin{array}{l}\text { Excited State } \\
\begin{aligned} 345->349 \\
346->350 \\
347->349 \\
347->350\end{aligned}\end{array}$ & 5: & $\begin{array}{c}\text { Singlet-A } \\
0.37171 \\
0.43037 \\
-0.38351 \\
-0.10922\end{array}$ & $2.1259 \mathrm{eV}$ & $583.20 \mathrm{~nm}$ & $\mathrm{f}=0.1272$ & $<\mathrm{S} * * 2>=0.000$ \\
\hline
\end{tabular}




\begin{tabular}{|c|c|c|c|c|c|c|}
\hline $\begin{array}{l}\text { Excited State } \\
345->349 \\
345->350 \\
346->349 \\
346->350 \\
347->350\end{array}$ & $6:$ & $\begin{array}{c}\text { Singlet-A } \\
0.35903 \\
-0.33665 \\
-0.32953 \\
-0.32239 \\
0.18013\end{array}$ & $2.1534 \mathrm{eV}$ & $575.75 \mathrm{~nm}$ & $\mathrm{f}=0.0717$ & $<\mathrm{S} * * 2>=0.000$ \\
\hline $\begin{array}{l}\text { Excited State } \\
\begin{aligned} 345->349 \\
345->350 \\
346->349 \\
346->350\end{aligned}\end{array}$ & $7:$ & $\begin{array}{l}\text { Singlet-A } \\
0.36391 \\
0.33267 \\
0.36601 \\
-0.33909\end{array}$ & $2.2104 \mathrm{eV}$ & $560.91 \mathrm{~nm}$ & $\mathrm{f}=0.0200$ & $<\mathrm{S} * * 2>=0.000$ \\
\hline $\begin{array}{l}\text { Excited State } \\
\qquad \begin{array}{l}345->350 \\
346->349\end{array}\end{array}$ & 8: & $\begin{array}{l}\text { Singlet-A } \\
0.50593 \\
-0.46391\end{array}$ & $2.3339 \mathrm{eV}$ & $531.23 \mathrm{~nm}$ & $\mathrm{f}=0.0073$ & $<\mathrm{S} * * 2>=0.000$ \\
\hline $\begin{array}{l}\text { Excited State } \\
\begin{array}{r}344->349 \\
344->350\end{array}\end{array}$ & 9: & $\begin{array}{l}\text { Singlet-A } \\
0.63968 \\
0.26162\end{array}$ & $2.7181 \mathrm{eV}$ & $456.15 \mathrm{~nm}$ & $\mathrm{f}=0.0129$ & $<\mathrm{S} * * 2>=0.000$ \\
\hline $\begin{array}{c}\text { Excited State } 1 \\
343->349 \\
344->349 \\
344->350\end{array}$ & 10: & $\begin{array}{c}\text { Singlet-A } \\
0.10743 \\
-0.24457 \\
0.63250\end{array}$ & $2.7289 \mathrm{eV}$ & $454.34 \mathrm{~nm}$ & $\mathrm{f}=0.0088$ & $<\mathrm{S} * * 2>=0.000$ \\
\hline
\end{tabular}




\section{References for Supporting Information}

1 Burla, M. C.; Caliandro, R.; Camalli, M.; Carrozzini, B.; Cascarano, G. L.; De Caro, L.; Giacovazzo, C.; Polidori, G.; Spagna, R. SIR2004: an improved tool for crystal structure determination and refinement. J. Appl. Cryst. 2005, 38, 381-388.

2 Sheldrick, G. M. Crystal structure refinement with SHELXL. Acta Crystallogr. C, Struct. Chem. 2015, $71,3-8$.

${ }^{3}$ (a) Wakita, K. Yadokari-XG, Software for Crystal Structure Analyses, Japan, 2001. (b) Kabuto, C.; Akine, S.; Nemoto, T.; Kwon, E. Release of software (Yadokari-XG 2009) for crystal structure analyses. J. Cryst. Soc. Jpn. 2009, 51, 218-224.

4 Nyokong, T.; Antunes, E. In Handbook of Porphyrin Science; Kadish, K. M., Smith, K. M., Guilard, R., Eds.; World Scientific: Singspore, 2010; Vol. 7, pp 247-357.

5 Maree, S. E.; Nyokong, T. Syntheses and photochemical properties of octasubstituted phthalocyaninato zinc complexes. J. Porphyrins Phthalocyanines 2001, 5, 782-792.

${ }^{6}$ (a) Becke, A. D. Density-functional exchange-energy approximation with correct asymptotic behavior. Phys. Rev. A 1988, 38, 3098-3100. (b) Becke, A. D. A new mixing of Hartree-Fock and local density-functional theories. J. Chem. Phys. 1993, 98, 1372-1377. (c) Becke, A. D. Density-functional thermochemistry. III. The role of exact exchange. J. Chem. Phys. 1993, 98, 5648-5652. (d) Lee, C.; Yang, W.; Parr, R. G. Development of the Colle-Salvetti correlation-energy formula into a functional of the electron density. Phys. Rev. B 1988, 37, 785-788.

7 Frisch, M. J.; Trucks, G. W.; Schlegel, H. B.; Scuseria, G. E.; Robb, M. A.; Cheeseman, J. R.; Scalmani, G.; Barone, V.; Mennucci, B.; Petersson, G. A.; Nakatsuji, H.; et al. Gaussian 09, Revision E.01; Gaussian, Inc., Wallingford CT, 2013.

8 (a) Bauernschmitt, R. d.; Ahlrichs, R. Treatment of electronic excitations within the adiabatic approximation of time dependent density functional theory. Chem. Phys. Lett. 1996, 256, 454-464. (b) Dreuw, A.; Head-Gordon, M. Single-reference ab initio methods for the calculation of excited states of large molecules. Chem. Rev. 2005, 105, 4009-4037. 